\title{
Neurod1 Is Essential for the Primary Tonotopic Organization and Related Auditory Information Processing in the Midbrain
}

\author{
Iva Macova, ${ }^{1,4 \star}$ Kateryna Pysanenko, ${ }^{2 \star}$ Tetyana Chumak, ${ }^{2}$ Martina Dvorakova, ${ }^{1,4}$ Romana Bohuslavova, ${ }^{1}$ Josef Syka, ${ }^{2}$ \\ Bernd Fritzsch, ${ }^{3}$ and Gabriela Pavlinkova ${ }^{1}$ \\ ${ }^{1}$ Institute of Biotechnology CAS, Vestec, Czechia 25250, ${ }^{2}$ Institute of Experimental Medicine CAS, Prague, Czechia 14220, ${ }^{3}$ Department of Biology, \\ University of Iowa, Iowa City, Iowa 52242, and ${ }^{4}$ Faculty of Science, Charles University, Prague, Czechia 12843
}

Hearing depends on extracting frequency, intensity, and temporal properties from sound to generate an auditory map for acoustical signal processing. How physiology intersects with molecular specification to fine tune the developing properties of the auditory system that enable these aspects remains unclear. We made a novel conditional deletion model that eliminates the transcription factor NEUROD1 exclusively in the ear. These mice (both sexes) develop a truncated frequency range with no neuroanatomically recognizable mapping of spiral ganglion neurons onto distinct locations in the cochlea nor a cochleotopic map presenting topographically discrete projections to the cochlear nuclei. The disorganized primary cochleotopic map alters tuning properties of the inferior colliculus units, which display abnormal frequency, intensity, and temporal sound coding. At the behavioral level, animals show alterations in the acoustic startle response, consistent with altered neuroanatomical and physiological properties. We demonstrate that absence of the primary afferent topology during embryonic development leads to dysfunctional tonotopy of the auditory system. Such effects have never been investigated in other sensory systems because of the lack of comparable single gene mutation models.

Key words: auditory pathway; cochlear nucleus; inferior colliculus; Neurod1 mutation; plasticity; sensory topographical map

Significance Statement

All sensory systems form a topographical map of neuronal projections from peripheral sensory organs to the brain. Neuronal projections in the auditory pathway are cochleotopically organized, providing a tonotopic map of sound frequencies. Primary sensory maps typically arise by molecular cues, requiring physiological refinements. Past work has demonstrated physiologic plasticity in many senses without ever molecularly undoing the specific mapping of an entire primary sensory projection. We genetically manipulated primary auditory neurons to generate a scrambled cochleotopic projection. Eliminating tonotopic representation to auditory nuclei demonstrates the inability of physiological processes to restore a tonotopic presentation of sound in the midbrain. Our data provide the first insights into the limits of physiology-mediated brainstem plasticity during the development of the auditory system.

\section{Introduction}

Sensory systems develop topographic maps of sensory inputs in nervous systems: the somatosensory system projects topograph-

Received Oct. 10, 2018; revised Nov. 17, 2018; accepted Dec. 5, 2018.

Author contributions: I.M. and K.P. wrote the first draft of the paper; J.S., B.F., and G.P. edited the paper; R.B., J.S., B.F., and G.P. designed research; I.M., K.P., T.C., M.D., R.B., and B.F. performed research; I.M., K.P., T.C., M.D., R.B., B.F., and G.P. analyzed data; B.F. and G.P. wrote the paper.

This work was supported by the (zech Science Foundation (17-04719S to G.P.), by BIOCEVCZ.1.05/1.1.00/ 02.0109 from the ERDF, by the Czech Academy of Sciences RV0: 86652036, by Charles University (GA UK 324615 to I.M. and GAUK 780216 to M.D.), and by the NIH (R01 AG060504 to B.F.). We thank Dr. K. Kandler for helpful comments on an earlier version of the paper, Dr. D. Šuta for help processing single-unit data, and A. Pavlinek for editing the paper.

The authors declare no competing financial interests.

*I.M. and K.P. contributed equally to this work.

Correspondence should be addressed to Gabriela Pavlinkova at Gabriela.Pavlinkova@ibt.cas.cz or Bernd Fritzsch at bernd-fritzsch@uiowa.edu. ical maps of the skin to the brainstem, midbrain, and cortex (Penfield and Boldrey, 1937; Renier et al., 2017), the olfactory system projects odor properties to a given olfactory glomerulus (Mombaerts, 1999; Gogos et al., 2000), the retina develops a topographical map in the midbrain (Sperry, 1963; Goodhill, 2007; Huberman et al., 2008), and vestibular, lateral line, and electroreceptive organs project to specific nuclei of the hindbrain and midbrain to establish computational maps (Krahe and Maler, 2014; Chagnaud et al., 2017). In the auditory system, cochlear sensory hair cells are connected to the brain by spiral ganglion (SG) neurons that are organized within the cochlea in an orderly fashion according to frequency, so called tonotopic organization, with high frequencies at the base and low frequencies at the apex 
(Rubel and Fritzsch, 2002; Muniak et al., 2016). This tonotopic (or cochleotopic) organization is maintained throughout the auditory pathways (Kandler et al., 2009). The formation of a tonotopic map requires the precise projection of the SG neuron afferents of the cochlea onto the first auditory nuclei of the hindbrain, the cochlear nuclei $(\mathrm{CN})$. In contrast to the bettercharacterized visual system or olfactory system, only some molecular mechanisms are known to lead to the cochleotopic mapping of spiral ganglion afferents onto the $\mathrm{CN}$ (Cramer and Gabriele, 2014; Goodrich, 2016; Yang et al., 2017) but it is unknown how much the initial cochleotopic map is physiologically refined (Marrs and Spirou, 2012). Developing second-order maps are highly plastic (Hubel et al., 1977; Renier et al., 2017) and convergence of multiple sensory organs can plastically reshape primary sensory maps as a compromise between activity and molecular cues (Constantine-Paton and Law, 1978; Elliott et al., 2015). Indeed, the auditory system is well known for having a high level of plastic changes throughout life (Syka, 2002; Eggermont, 2017) but how embryonic development affects and possibly limits these later plastic changes remains unclear because of the lack of models (Kral et al., 2016) beyond simply removing parts of the cochlea (Harrison, 2016).

In the formation of the primary tonotopic map, several factors apparently define the precision of some SG neuron projections (Cramer and Gabriele, 2014; Lu et al., 2014; Goodrich, 2016; Yang et al., 2017). The basic helix-loop-helix (bHLH) gene Neurod1 (Liu et al., 2000) was shown to be essential for inner ear neuronal development as well as normal growth of the cochlea. Subsequent work on mutants null for Neurod1 showed retention of some sensory neurons using specific neuronal tracing techniques (Kim et al., 2001). NEUROD1 cross-regulates other transcription factors in neurons and hair cells, leading to the transformation of some neurons to intraganglionic hair cells, and transformation of some outer hair cells to inner hair cells (Jahan et al., 2010b). In addition, deletion of Neurod 1 leads to gross projection mapping errors of the few remaining neurons (Jahan et al., 2010a) that go beyond those described in other primary sensory system (Huberman et al., 2008). In previously generated mutants, Neurod 1 deletion occurs both in the ear and the central auditory nuclei, which limits SG neuronal viability and hampers physiological assessment of the wiring defects (Gurung and Fritzsch, 2004; Fritzsch et al., 2006; Jahan et al., 2010a).

We therefore generated a novel mutant with a conditional deletion of Neurod 1 only in the ear to spare many SG neurons and to retain Neurod1 expression in the auditory nuclei and auditory midbrain. We show here how a shortened and nearly overlapping cochleotopic projection from SG neurons to the $\mathrm{CN}$ is expanded across the entire inferior colliculus (IC), affecting the frequency, intensity, and temporal processing of the central auditory system of adult mice at the physiological and behavioral level. Unique to our study are the consequences of compressing the unsegregated and disorganized peripheral projection map of SG neurons onto the tonotopic organization of the central auditory pathways. This type of disorganization of a neural map of the sensory periphery is nearly impossible to achieve with other sensory systems that would require, for example, trigeminal neurons to the face to also innervate the foot or retina ganglion neurons to connect to both eyes and the brain.

\section{Materials and Methods}

Animals

All experiments using animals were performed according to protocols approved by the Animal Care and Use Ethics Committee of the Institute of Molecular Genetics, Czech Academy of Sciences. The experimental mice were housed in a controlled environment ( $12 \mathrm{~h}$ light/dark cycles) with ad libitum access to food and water. All experiments were performed with littermates (males and females) crossbred from two transgenic mouse lines: floxed Neurod1 (Neurod1 ${ }^{\text {loxP/loxP }}$; Goebbels et al., 2005) and Islet1-cre (Isl1-cre; Isl1 $\left.{ }^{\mathrm{tm} 1(\mathrm{cre}) \mathrm{Sev} / \mathrm{I}}\right)$ from The Jackson Laboratory. Breeding pairs contain a mouse with two floxed Neurod 1 alleles (Neurod $1^{\text {loxP/loxP }}$ ) and a mouse with one floxed Neurodl allele together with one Isl1-cre allele $\left(\right.$ Isl $1^{\text {cre/+}} ;$ Neurod $\left.1^{\text {loxP/+ }}\right)$. Genotyping was performed by PCR on tail DNA. The specific primers used were the following: Isl1-cre F 5'-GCC TGC ATT ACC GGT CGA TGC AAC GA-3' and Isl1-cre R 5' -GTG GCA GAT GGC GCG GCA ACA CCA TT-3' with a 700 bp product; Neurod1 F 5'-ACC ATG CAC TCT GTA CGC ATT-3' and Neurod1 R 5'-GAG AAC TGA GAC ACT CAT CTG-3' with a 400 bp product for the WT allele or $600 \mathrm{bp}$ for the floxed allele. Heterozygous mice $\mathrm{Isl}^{\mathrm{cre} /+}$; Neurod1 $1^{\text {loxP/+ }}\left(\right.$ HET) were comparable to the control mice $\left(I s l 1^{+/+}\right.$; Neurod $1^{\text {loxP/loxP }}$ or $I s l 1^{+/+} ;$Neurod $\left.1^{\text {loxP/+}}\right)$ without any detectable morphological and functional differences. Neurod1cKO offspring were recovered at expected Mendelian ratios from E9.5 to P0 birth (123 litters collected and genotyped: 234 Neurod1cKO: 260 HET: 534 control offspring). The sex ratio of Neurod 1cKO mice at weaning showed the same representation of males and females as the control mice.

\section{Morphology of the cochlea, cochlear nuclei, and IC}

$X$-gal staining. The mouse line Isll-cre was bred with R26R-lacZ (Gt(ROSA)26Sor ${ }^{\text {tm } 1 \text { Sor }}$; The Jackson Laboratory) and animals carrying both loci were subjected to X-gal staining (Dvorakova et al., 2016).

Lipophilic dye tracing. We studied the pattern of innervation in whole or dissected ears using lipophilic dye tracing in aldehyde fixed tissue as previously described (Fritzsch et al., 2016a). At least three mutants and similar numbers of control littermates of both sexes were shipped to the University of Iowa and used for different stage (E13.5, E14.5, E16.5, 6 at E18.5, P0, P3 and 6 at P7). We inserted filter strips loaded with different colored lipophilic dyes into the cochlear apex, base, vestibular end organs, cochlear/vestibular nuclei of the brainstem around rhombomere 5 to label afferents, and into rhombomere 4 near the midline to label facial motoneurons/efferents to the ear (Simmons et al., 2011). After allowing appropriate time for diffusion of the lipophilic tracer (between 48 and $120 \mathrm{~h}$ ), we prepared the ears as whole mounts, mounted with glycerol on a glass slide, using appropriate spacers to avoid distortion, and imaged them using a Leica SP8 confocal microscope. Image stacks were collected and single images or sets of stacks were obtained to provide detailed information about the progressive development and loss of ear innervation over time. Selected ears were further dissected to reveal the detailed innervation of the flat mounted sensory epithelia. Images were compiled into plates to show the most pertinent details using Corel Draw. Only general image modifications, such as contrast or brightness adjustments, were used to enhance the visual appeal without affecting the scientific content.

Immunohistochemistry and morphological evaluations. For whole mounts, dissected tissues were fixed in $4 \%$ paraformaldehyde (PFA). For vibratome sections, $4 \%$ PFA-fixed samples were embedded in $4 \%$ agarose gel and sectioned at $80 \mu \mathrm{m}$ on a Leica VT1000S vibratome. The primary antibodies used were rabbit anti-Myo7a (1:500; 25-6790, Proteus BioSciences), rabbit or mouse anti-NeuN (1:500, ab177487, Abcam; or 1:100, MAB377, Merck), mouse anti-acetylated $\alpha$-tubulin (1:400; T6793, Sigma-Aldrich), mouse anti-tubulin $\beta 3$ (Tuj1; 1:500; 801202, BioLegend), rabbit anti-neurofilament 200 (1:200; N4142, SigmaAldrich), rabbit anti-Proxl (1:500; 925201, BioLegend), mouse antiVGLUT1 (1:200; MAB5502, Merck), rabbit anti-parvalbumin (1:2000; ab11427, Abcam), rabbit anti-calretinin (1:100; sc-50453, Santa Cruz Biotechnology), goat anti-prestin (1:50; sc-22692, Santa Cruz Biotechnology), goat anti-Neurod1 (1:100; sc-1084, Santa Cruz Biotechnology), 
mouse anti-Islet1 (1:50; Developmental Studies Hybridoma Bank 39.3F7 was deposited to the DSHB by T. M. Jessell/S. Brenner-Morton), and mouse anti-C-terminal binding protein 2 (CtBP2; 1:200; 612044, BD Biosciences). The secondary antibodies used were AlexaFluor 488 AffiniPure Goat Anti-Mouse IgG (1:500; 115-545-146, Jackson ImmunoResearch), AlexaFluor 594 AffiniPure Goat Anti-Rabbit (1:500; 111585-144, Jackson ImmunoResearch), and DyLight488-conjugated AffiniPure Mouse Anti-Goat IgG (1:500; 205-485-108, Jackson ImmunoResearch). Nuclei were stained by Hoechst 33258 (1:2000; 861405, Sigma-Aldrich). Samples were mounted in Aqua-Poly/Mount (18606, Polysciences) or in prepared Antifade medium and images were taken on Zeiss LSM 5 DUO, Zeiss LSM 880, or Leica SPE confocal microscopes. ImageJ and ZEN software were used for image processing.

For neuron quantification, neurons were counted in all NeuN/Tuj1 stained vibratome sections containing a SG using the "Cell Counter" ImageJ plugin, as described previously (Bohuslavova et al., 2017). Briefly, the total number of neurons from all sections per individual cochlea was determined ( $n=3 /$ genotype/age). The mean number of neurons in control mice represented $100 \%$ of the SG neurons. To determine the length of the cochleae, individual adult cochleae were flat-mounted with the sensory epithelium facing up and the entire length of the cochlear duct from the hook region along the basilar membrane was measured using the "Measure line" ImageJ plugin ( $n=3 /$ genotype). The volume of the $\mathrm{CN}$ and IC was established by analyzing parallel serial equally spaced sections through the brain. Eighty micrometer coronal vibratome sections were prepared by sectioning five brains of control and five brains of mutant mice. The areas of the left and right $\mathrm{CN}$ and IC were determined in each section using ImageJ, and the volume of the organs was calculated. Volumes of paired organs were normalized to body weight. The quantification of neuron soma size was done using the lipophilic tracer dye labeled samples. The area of neuron somas ( 80 neurons/2 mutants) was determined in image stacks using ImageJ. Data are provided as mean \pm SD.

c-Fos staining. Auditory stimulation was described previously (Karmakar et al., 2017). For each experiment, two 2-month-old mice (control and Neurod1cKO pair) were placed in a small wire cage under a speaker in a soundproof room. These animals were kept in silence for $1 \mathrm{~h}$ and then $15 \mathrm{kHz}$ tone pips at $75 \mathrm{~dB}$ sound pressure level (SPL) followed for 90 min. Immediately after sound exposure, mice were transcardially perfused by $4 \%$ PFA and brains were dissected and fixed in 4\% PFA for $1 \mathrm{~h}$. Tissues were mounted into $4 \%$ agarose in PBS and $80 \mu \mathrm{m}$ coronal sections were cut on a Leica VT1000S vibratome. Sections were blocked in $2.5 \%$ normal goat serum, $0.5 \%$ Tween and $0.1 \%$ Triton in PBS. To detect activated neurons in the $\mathrm{CN}$, we used primary rabbit anti-c-Fos antibody (1:5000; PC38, Calbiochem) and secondary goat anti-rabbit antibody conjugated by AlexaFluor 594 (1:500; 111-585-144, Jackson ImmunoResearch). Stained sections were mounted in Antifade medium and pictures were taken on a Zeiss LSM 880. c-Fos ${ }^{+}$neurons in the CN were counted using Cell Counter (ImageJ) and statistics were made using GraphPad software (unpaired $t$ test, $n=4$ /genotype).

\section{Hearing function evaluation}

Auditory brainstem response (ABR) and distortion product otoacoustic emission (DPOAE) tests were performed on mice as described previously (Chumak et al., 2016). Briefly, for ABR recording, responses to tone bursts ( $3 \mathrm{~ms}$ duration, $1 \mathrm{~ms}$ rise/fall times, frequencies of $2,4,8,16,32$, and $40 \mathrm{kHz}$ ) and clicks of different intensity were recorded. The response threshold to each frequency was determined as the minimal tone intensity that still evoked a noticeable potential peak in the expected time window of the recorded signal. Click-evoked ABR responses were used to analyze the amplitude of single ABR waves and the latency of positive ABR peaks I to IV. For comparison of amplitude-intensity functions, the amplitude of a single ABR wave was calculated as the peak to peak interval and normalized in relation to the wave amplitude at $90 \mathrm{db}$ SPL because of significant differences in ABR wave amplitude between the groups. For DPOAE tests, cubic (2 F1-F2) distortion product otoacoustic emissions over a F2 frequency range from 4 to $38 \mathrm{kHz}$ were recorded (control, $n=12$; NeurodlcKO, $n=8$ ).
Table 1. Number of animals used

\begin{tabular}{lll}
\hline & No. of animals & \\
\cline { 2 - 3 } Experimental approach & WT & Neurod7cK0 \\
\hline Histology of the inner ear & 39 & 38 \\
Histology of the brain & 20 & 17 \\
Dye tracing & 27 & 27 \\
Physiology & 30 & 25 \\
\hline
\end{tabular}

\section{Behavioral tests}

In the behavioral tests, eight mice were used from both experimental groups (control and NeurodlcKO) at the age of 2-3 months. All behavioral tests were performed in a sound-attenuated chamber (Coulbourn Habitest, model E10-E21) located in a soundproof room. During the testing procedure, the mouse was confined to a small wire mesh cage on a motion-sensitive platform. The animal's reflex movements were detected and transduced by a piezoelectric accelerometer. The amplified voltage signal was acquired and processed using a TDT system III with Enhanced Real-Time Processor RP2.1 (Tucker Davis Technologies) and custom-made software programmed in MATLAB. The startle responses were evaluated in a $100 \mathrm{~ms}$ window beginning at the onset of the startle stimulus. The magnitude of the acoustic startle reflex was given by the maximal peak-to-peak amplitude of transient voltage occurring in the response window. Acoustic startle stimuli (tone pips or noise bursts) and prepulse stimuli were generated by the TDT system and presented via a loudspeaker (SEAS, 29AF/W) placed $12 \mathrm{~cm}$ above the platform inside the chamber. Stimulus presentation and data acquisition were controlled by a custom-made application in MATLAB. The acoustic startle reflex (ASR; a transient motor response to an intense, unexpected stimulus) was used as an indicator of the behavioral responsiveness to sound stimuli. The ASRs to 4, 8, 16, and $32 \mathrm{kHz}$ tone pips and white noise (WN) bursts ( $50 \mathrm{~ms}$ duration, $3 \mathrm{~ms}$ rise/fall times, varying intensity levels) were recorded. Each test session contained the following: a baseline trial $(-10$ dB SPL stimulus intensity) and 13 startle stimuli of different intensities $(50,55,60,65,70,75,80,85,90,100,110,115$, and $120 \mathrm{~dB}$ SPL). The intertrial interval varied from 15 to $50 \mathrm{~s}$.

In the prepulse inhibition procedure, three different trial types were used: a baseline trial without any stimulus, an acoustic startle pulse alone (WN at $110 \mathrm{~dB}$ SPL, $50 \mathrm{~ms}, 3 \mathrm{~ms}$ rise/fall times), and a combination of the prepulse and startle pulse. The interstimulus interval between the prepulse and the startle stimulus was set to $50 \mathrm{~ms}$; each of the trial types was presented three times. The intertrial interval was randomized and varied from 15 to $30 \mathrm{~s}$. The efficacy of the prepulse inhibition (PPI) of the ASR was expressed as an ASR ratio in percentage: 100\% corresponds to the amplitude of the ASR without a prepulse; smaller values of the ASR ratio indicate greater PPI. As a prepulse we used: either (1) WN bursts or tone pips (50 ms duration, $3 \mathrm{~ms}$ rise/fall time) at frequencies of 4,8 , and 16 $\mathrm{kHz}$ at increasing intensities; or (2) a gap in the background WN of low intensity ( $65 \mathrm{~dB}$ SPL). Both, when presented before the startle stimulus, are expected to decrease the amplitude of the startle response that followed.

\section{Vestibular tests}

To evaluate the motor coordination of the mice from both strains (control, $n=4$; Neurod1cKO, $n=3$ ), we used three stable rods of varying thickness and length (rod 1: $25 \mathrm{~mm}$ diameter, $50 \mathrm{~cm}$ distance from the open end to the end line; rod 2: $20 \mathrm{~mm}, 35 \mathrm{~cm}$ to the end line; and rod 3 : $15 \mathrm{~mm}$ diameter, $35 \mathrm{~cm}$ to the end line). Two times were measured: orientation time, time taken to turn $180^{\circ}$ from the starting position toward the shelf; and transit time, the time taken to travel to the end line (Deacon, 2013). For statistical purposes, we set the maximal test time at $300 \mathrm{~s}$. If the mouse fell off a rod, the maximal score (300 s) was assigned for that particular rod. All tests were performed in an anechoic soundproof room and acoustic stimuli were delivered in free-field conditions via a two-driver loudspeaker system (Selenium 6W4P woofer and RAAL70-20 tweeter). Four types of trials were used: silence, the presence of a continuous broad band noise with intensity $80 \mathrm{~dB}$ SPL and presence of a $600 \mathrm{~ms}$ series of $100 \mu$ s clicks at an intensity of $70 \mathrm{~dB}$ SPL with 

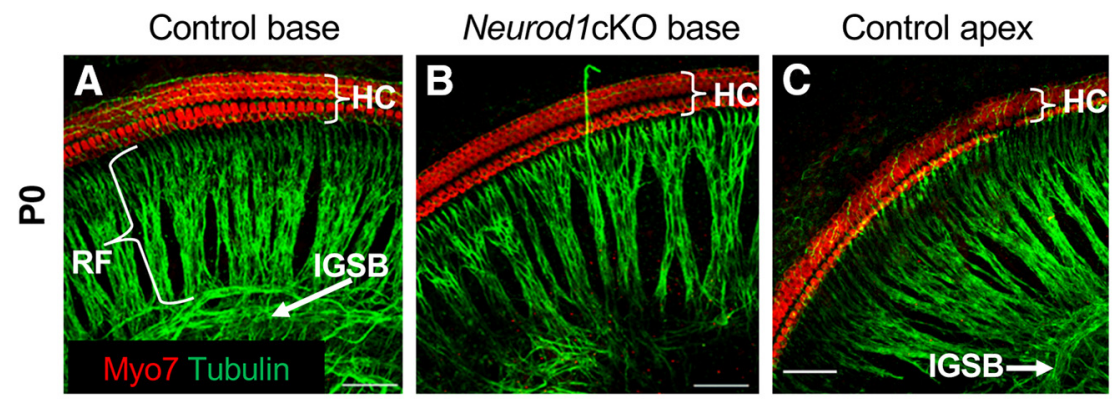

\section{Neurod1cKO apex}
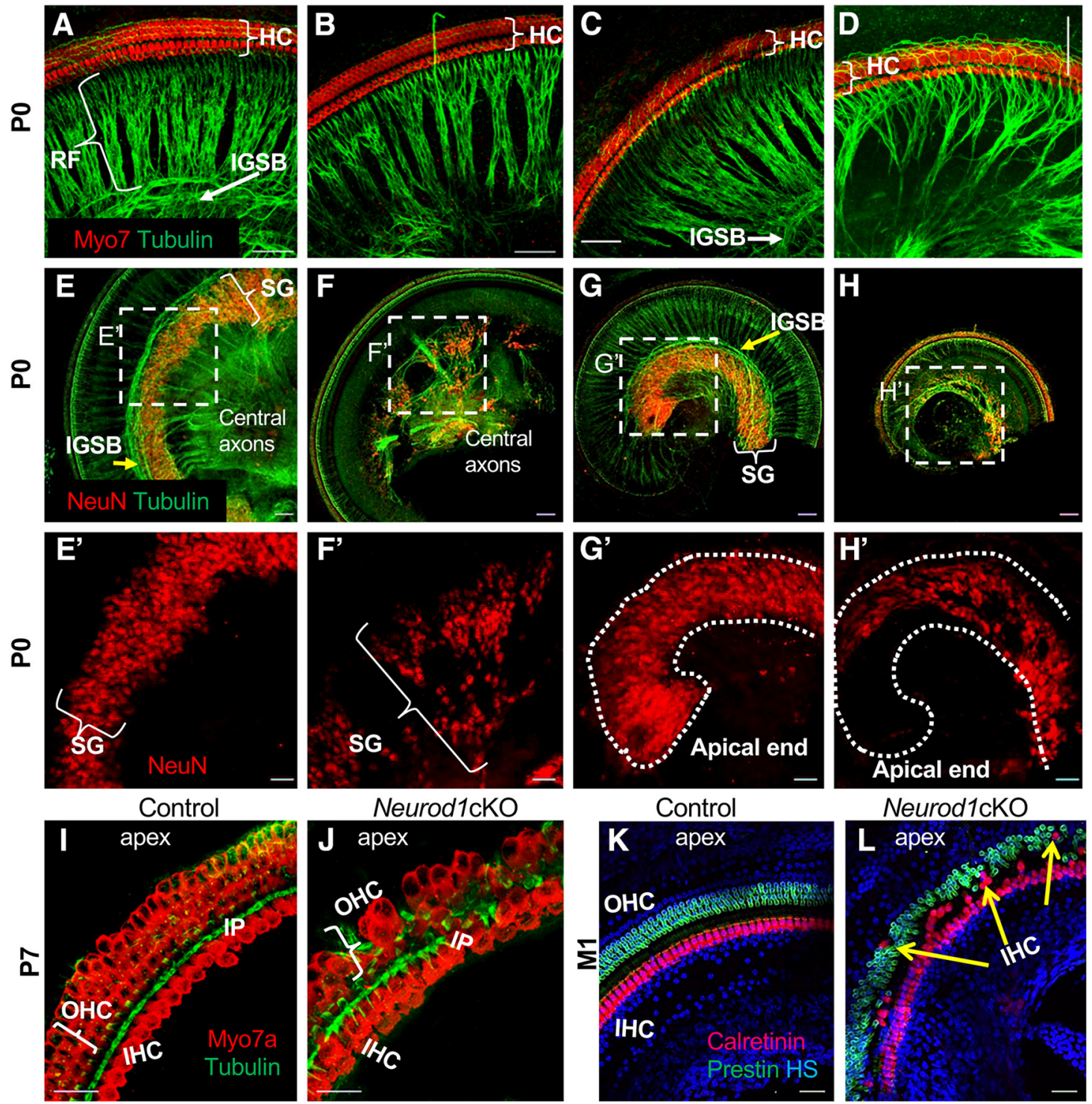

Neurod1cKO
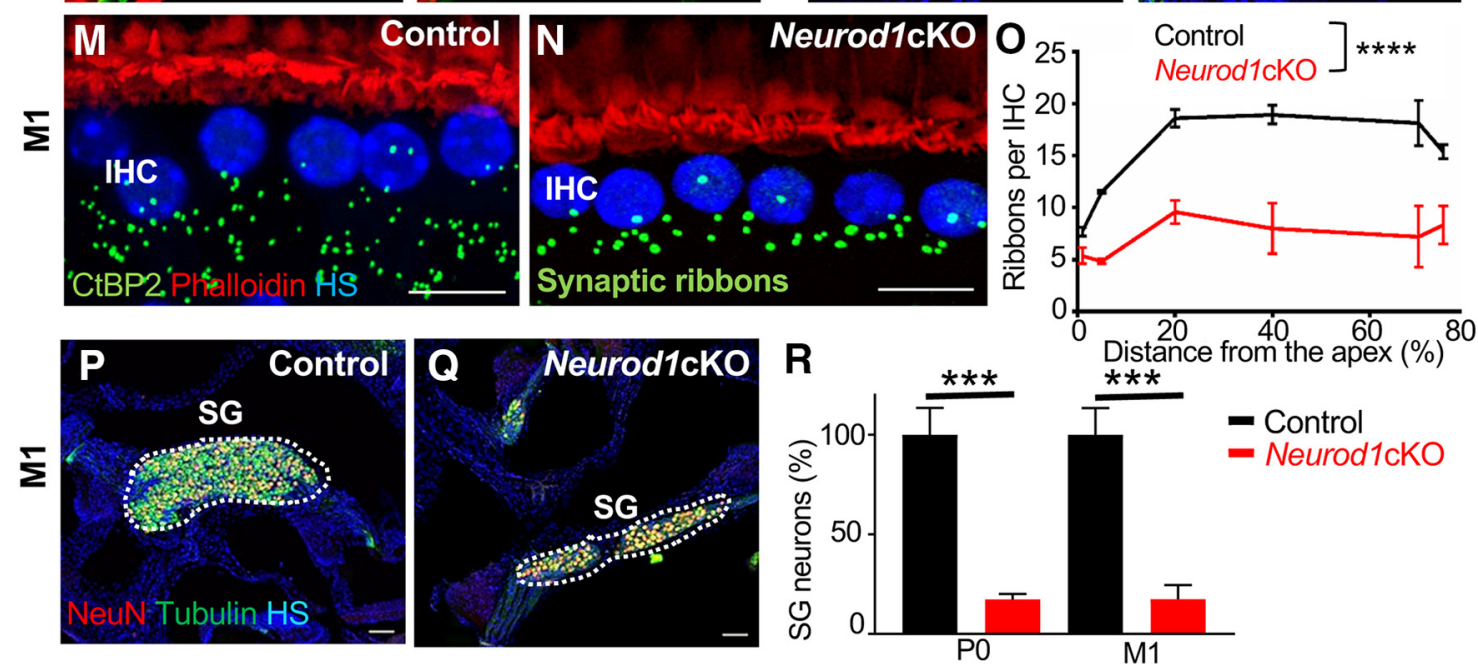

Figure 1. Morphology of the Neurod 7 cKO inner ear is altered. $\boldsymbol{A}-\boldsymbol{D}$, Whole-mount immunostaining with anti-Myo7a [a marker of hair cells (HCs)] and anti-acetylated $\alpha$-tubulin (nerve fibers) antibodies shows subtle changes in a number of radial fibers (RFs), lack of the intraganglionic spiral bundle (IGSB; arrow), noticeable disorganization of RFs, and overshooting fibers in the apex of Neurod7cKO cochlea (C, D). Scale bar, $50 \mu \mathrm{m}$. $\boldsymbol{E}-\boldsymbol{H}$, Whole-mount immunostaining of SG neurons (anti-NeuN, a neuronal soma marker) and innervation shows reduction and disorganization of SG neurons in mutants. Scale bar, $50 \mu \mathrm{m} . \boldsymbol{E}^{\prime}-\boldsymbol{H}^{\prime}$, Higher-magnification images demonstrate a decreased number and altered distribution of $S G$ neurons. Scale bar, $20 \mu \mathrm{m} . \boldsymbol{I}, \boldsymbol{J}, \mathrm{Imm}$, for My07a and for $\beta$ tubulin (a marker of inner pillar supporting cells) shows the disorganization of OHCs (red) and inner pillar cells (IPs; green) in the apex in Neurod1 cKO compared with the organ of Corti of control littermates at P7. Scale bar, $20 \mu \mathrm{m}$. HS, Hoechst nuclear staining. $\boldsymbol{K}, \boldsymbol{L}$, Immunohistochemistry for prestin (a marker for OHC) and calretinin (Figure legend continues.) 
interstimulus intervals linearly decreasing and increasing from 25 to 6.6 $\mathrm{ms}$ and back to $25 \mathrm{~ms}$.

\section{Extracellular recording of the neuronal activity in the IC}

For extracellular recording, Neurod1cKO ( $n=9 ; 432$ units from the IC) and control mice ( $n=10 ; 480$ units) were tested. The surgery and extracellular recording in the IC were performed in mice anesthetized with 35 $\mathrm{mg} / \mathrm{kg}$ ketamine (Calypsol, $50 \mathrm{mg} / \mathrm{ml}$ ) and $6 \mathrm{mg} / \mathrm{kg}$ xylazine (Xylapan, 20 $\mathrm{mg} / \mathrm{ml}$ ) in saline via intraperitoneal injection. Approximately every hour, additional subcutaneous injections of one-half of the original dose of the anesthetics were administered to keep a sufficient level of anesthesia. Basic reflexes ( pedal reflex or eye blink reflex), respiratory rate, and heart rate, were monitored. For access to the IC, an incision was made through the skin of the skull and underlying muscles were retracted to expose the dorsal cranium. A holder was glued to the skull and small holes were drilled over both ICs. Neuronal activity (multiple units) in the IC was recorded using a 16-channel, single shank probe (NeuroNexus Technologies) with $50 \mu \mathrm{m}$ between the electrode spots. The signal obtained from the electrode was amplified 10000 times, bandpass filtered over the range of $300 \mathrm{~Hz}$ to $10 \mathrm{kHz}$ and processed by a TDT System III (Tucker Davis Technologies) using an RX5-2 Pentusa Base Station. The data were recorded and processed in BrainWare software (Jan Schnupp, Tucker Davis Technologies) for artifact rejection and separation of single units on the basis of spike-shape clustering and then analyzed with custom software based on MATLAB (MathWorks). The recorded data were processed and analyzed with custom software based on MATLAB. The stimulation signals were generated using a TDT System III with the RP 2.1 Enhanced Real-Time Processor. Acoustic stimuli were delivered in free-field conditions via a two-driver loudspeaker system (Selenium $6 \mathrm{~W} 4 \mathrm{P}$ woofer and RAAL70-20 tweeter) placed $70 \mathrm{~cm}$ in front of the animal's head.

Frequency-intensity mapping. To determine the neuronal receptive fields, pure tones (frequency $2-40 \mathrm{kHz}$ with $1 / 8$ octave step, $60 \mathrm{~ms}$ duration, $5 \mathrm{~ms}$ rise/fall times, various intensity with $5 \mathrm{~dB}$ step) were presented in a random order, each stimulus appearing three times. A discrete matrix corresponding to the response magnitude evoked by each of the frequency-intensity combinations was thereby obtained, smoothed using cubic spline interpolation and used for extraction of the basic parameters: the excitatory response threshold (the lowest stimulus intensity that excited the neuron, measured in $\mathrm{dB}$ SPL), the characteristic frequency $(\mathrm{CF})$, the frequency with the minimal response threshold (measured in $\mathrm{Hz}$ ), and the bandwidth of the excitatory area 10, 20, and $30 \mathrm{~dB}$ above the excitatory threshold, expressed by quality factor $\mathrm{Q}(\mathrm{Q}=\mathrm{CF} /$ bandwidth).

Two-tone stimulation. To detect inhibitory areas, a two-tone stimulation was used. Pure tone at the neuron's CF fixed $10 \mathrm{~dB}$ above the threshold at CF and pure tone pips of variable frequency and intensity, analogous to those used for the excitatory area mapping, were simultaneously presented. Similar to frequency-intensity mapping, a twodimensional matrix was obtained, and the presence of the low- and high-frequency sideband inhibitory areas was evaluated.

Rate intensity function of the IC neurons. Neuronal responses to broadband noise (BBN) bursts of variable intensity (10 dB steps, 50 repetitions) were used to construct the rate intensity function (RIF). On each RIF, two points of interest were defined: R10, describing the starting

$\leftarrow$

(Figure legend continued.) (a marker for IHCs) shows trans-differentiated IHCs instead of OHCs (arrows) in the apex of the mutant cochlea at 1 month of age (M1). Scale bar, $20 \mu \mathrm{m} . M$, $\boldsymbol{N}$, Confocal analysis of immunostaining for a synaptic ribbon protein (CtBP2) in the IHC area shows a reduction of ribbons in mutant adult mice compared with the controls. Phalloidin labels F-actin in stereocilia of hair cells. HS, Nuclear staining Hoechst. Scale bar, $10 \mu \mathrm{m}$. 0, Quantification of ribbon synapses per IHC area along the tonotopic axis. Data are expressed as the mean \pm SEM; $n=3$; two-way ANOVA. ${ }^{* * *} p<0.0001$. $\boldsymbol{P}, \boldsymbol{Q}$, Immunohistochemistry for NeuN in SG neurons at M1, the dotted line indicates the boundaries of SG in the vibratome sections of the cochlea. Scale bar, $50 \mu \mathrm{m}$. $\boldsymbol{R}$, Quantification of SG neurons in the control and Neurod 1 CKO cochlea at P0 and M1. Data are represented as the mean \pm SEM; $n=3 /$ genotype/ age. Two-tailed unpaired $t$ test. ${ }^{* * *} p<0.001$. point of the RIF's rise, and R90, describing the RIF's transition to the saturated region. A $100 \%$ scale was assigned to the neuron's total range of response amplitudes, with $0 \%$ corresponding to spontaneous activity and $100 \%$ corresponding to its maximum response magnitude. The two points of interest, R10 and R90, correspond to 10 and $90 \%$ of this scale, respectively. On each RIF, two points of interest were defined: R10, describing the starting point of the RIF's rise, and R90, describing the RIF's transition to the saturated region (Bures et al., 2010). The RIF was qualified as saturating if the response magnitude within the top $10 \mathrm{~dB}$ interval of the highest stimulus intensities was flat $( \pm 10 \%)$. The RIF was qualified as non-monotonic if the response magnitude at the highest stimulus intensity was smaller than the maximum response magnitude by $>20 \%$. The remaining RIFs were qualified as strictly monotonic. RIFs were further used for evaluating the following parameters: the percentage of saturating, non-monotonic, and strictly monotonic RIFs; the sound pressure level (S10) corresponding to point R10; the relative response at $\mathrm{R} 10$; the dynamic range (DR) of the RIF: $\mathrm{DR}=\mathrm{S} 90-\mathrm{S} 10$ ); and the maximum response magnitude. Spontaneous activity of the IC neurons was determined at the $0 \mathrm{~dB}$ SPL BBN stimulation. A Fisher's exact test was used to examine the relationship between mutant and control neurons with a certain RIF.

Temporal properties of the IC neurons. Two types of stimuli were used: (1) a $600 \mathrm{~ms}$ series of $100 \mu$ s clicks at an intensity of $70 \mathrm{~dB}$ SPL for control and $80 \mathrm{~dB}$ SPL for Neurod $1 \mathrm{cKO}$ mice with interstimulus intervals linearly decreasing and increasing from 25 to $6.6 \mathrm{~ms}$ and back to $25 \mathrm{~ms}$, and (2) trains of five clicks at an intensity of $70 \mathrm{~dB}$ SPL for control and $80 \mathrm{~dB}$ SPL for Neurod1cKO mice with various interclick intervals $(100,50,30,20$, 15,10 , and $5 \mathrm{~ms}$ ). In the case of the long changing clicks stimulus, we calculated the percentage of the clicks in the train to which the neurons responded in the time window that started $5 \mathrm{~ms}$ after the click and lasted $5 \mathrm{~ms}$. For trains of five clicks with different interspike intervals we computed the vector strength (VS) values along with the Rayleigh statistics that were computed for each spike pattern; only responses with Rayleigh statistics of least 5.991 were considered as significantly phase-locking (Zhou and Merzenich, 2008). The VS quantifies how well the individual spikes are synchronized (phase-locked) with a periodic signal.

\section{Experimental design and statistical analysis}

All comparisons were made between animals with the same genetic background, typically littermates. The number of mice used for different analyses was as follows:

The number of samples $(n)$ for each comparison can be found in the individual method descriptions and are given in the corresponding figures. Note that for histology and dye tracing we used the left and right ears as independent samples, doubling the total given in Table 1. Phenotyping and data analysis were performed blind to the genotype of the mice. All values are presented either as the mean \pm SD or SEM. For statistical analysis, GraphPad Prism software was used. To assess differences in the mean, two-sided Student's $t$ tests, one-way or two-way ANOVA with Bonferroni's multiple-comparison test, multiple $t$ test with Holm-Sidak comparison method, $\chi^{2}$ test, and unpaired two-tailed $t$ tests were used. Significance was determined as ${ }^{\star} p<0.05,{ }^{* *} p<0.01$, or ${ }^{* * *} p<0.001$. Complete results of the statistical analyses, including exact $p$ values are included in the figure legends.

\section{Results}

Neurod1-deficient mice retain many SG neurons

Neurod1 was eliminated specifically in the inner ear by crossing Neurod $1^{\text {loxP/loxP }}$ mice (Goebbels et al., 2005) with Islet $1^{\text {cre }}$ mice (Dvorakova et al., 2016; Neurod1cKO). Neurod1cKO mice are viable without any obvious abnormal motor activity behavior that would indicate major defects in the vestibular system. To evaluate the cochlear phenotype of Neurod1cKO mutants, we stained cochlear whole mounts with antibody to Myo7a, a hair cell marker. The organization of the organ of Corti of mutants was comparable to controls, with three rows of outer hair cells (OHCs) and one row of inner hair cells (IHCs; Fig. 1A-D). In contrast to previous work showing that all (Liu et al., 2000) or 

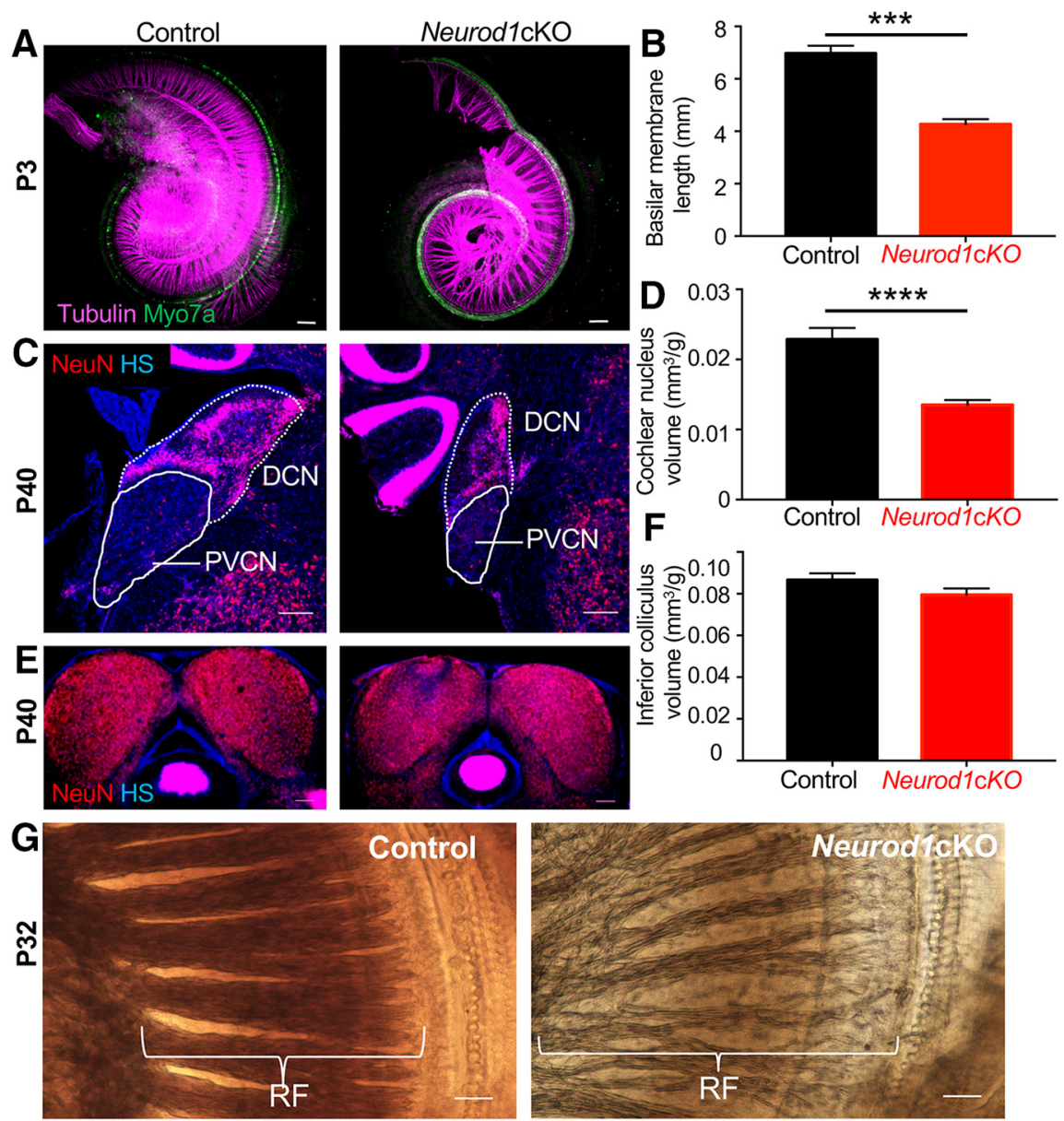

H

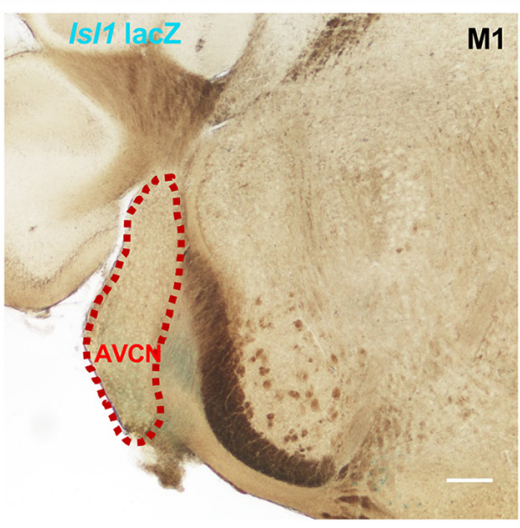

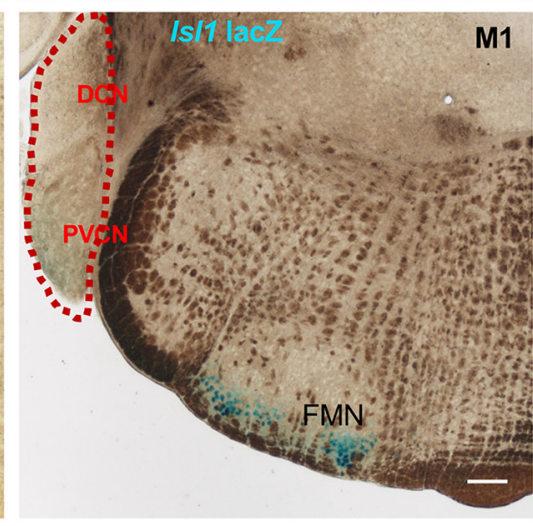

Figure 2. Deletion of Neurod1 affects the size of the components of the auditory pathways. $\boldsymbol{A}, \boldsymbol{B}$, Representative image of a whole-mount of control and Neurod1cK0 cochlea at P3. Hair cells labeled with anti-Myo7a (green) and neuronal fibers with anti- $\beta$-tubulin (magenta). Note a reduced density of radial fibers in Neurod1cK0 cochlea. Scale bar, $100 \mu \mathrm{m}$. Quantification of cochlear length at 4 weeks of age $(n=3)$. C, Sections of the CN immunostained with anti-NeuN (red) of adult control and Neurod1cK0, showing the DCN and PVCN. The line indicates the boundaries of the CN. HS, Nuclear staining Hoechst. Scale bar, 200 $\mu \mathrm{m}$. D, Quantification of the volume of the adult $\mathrm{CN}$ normalized to body weight $(n=5) . \boldsymbol{E}, \boldsymbol{F}$, Immunostaining of coronal brain sections for NeuN and quantification of the volume of the adult control and Neurod1cKO IC, normalized to body weight $(n=5)$. Scale bar, $200 \mu \mathrm{m}$. Data $(\boldsymbol{B}, \boldsymbol{D}, \boldsymbol{F})$ are the mean \pm SEM. Two-tailed unpaired $t$ test. ${ }^{* * *} p<0.001,{ }^{* * * *} p<0.0001 . \mathbf{G}$, In the adult cochlea, decreased density and increased length of radial fibers (RFs) stained by 0 s04 in Neurod1 cK0 are noticeable compared with the control. Scale bar, $100 \mu \mathrm{m}$. $\boldsymbol{H}$, Using a LacZ reporter (R26R mouse line), Isl1-cre-mediated $\beta$-galactosidase reporter expression shows no Cre recombination in the $\mathrm{CN}$ in the brain sections. The dotted line indicates the boundaries of the CN. Cre recombination is detected by the LacZ reporter in the facial motor nucleus (FMN). Scale bar, $200 \mu \mathrm{m}$.

nearly all SG neurons degenerate in different Neurod1 mutants (Kim et al., 2001; Jahan et al., 2010a), we detected dense radial fibers and SG neurons in the cochlea of NeurodlcKO (Fig. 1A$\left.D, E-H, E^{\prime}-H^{\prime}\right)$. However, there were noticeable abnormalities associated with aberrant innervation and migration of neurons in the cochlea, e.g., elongated and reduced radial fibers, increased spacing between radial fiber bundles, no intraganglionic spiral bundle formed by efferent axons, spread out and missing SG neurons, disorganized central axons, and complete absence of SG at the apical end. In the apex of NeurodlcKO, radial fiber bundles were noticeably disarranged with crossing fibers (Fig. 1D) and the epithelium was disorganized with missing OHCs, transdifferentiated OHCs into IHCs, and disarranged $\beta$-tubulin ${ }^{+}$supporting pillar cells (Fig. $1 I-L$ ). This sensory epithelium phenotype of trans-differentiation of some OHCs into IHCs was comparable to previous reports on different Neurod1 deletion mutants (Liu et al., 2000; Kim et al., 2001; Jahan et al., 2010a). We also directly assessed the synapses between auditory nerve terminals and IHCs by immunostaining the cochlear sensory epithelium for CtBP2, a major component of presynaptic ribbons in the adult animals (Chumak et al., 2016). Ribbon counts showed an average reduction of $58 \%$ in Neurod1cKO compared with controls, indicating a reduction of IHC afferent ribbon synapses (Fig. $1 M-O$ ). The total number of SG neurons positioned inside the Neurod1cKO cochlea was reduced at P0 by $80 \%$ compared with control littermates but the number of surviving SG neurons was maintained during postnatal development up to adulthood (Fig. $1 P-R)$.

\section{Neurod 1cKO mice have a shortened cochlea, smaller cochlear nuclei, normal size of the IC, and altered ABR} The dissected cochleae of control and Neurod1cKO mice were mounted, imaged in a confocal microscope and the length of the organ of Corti was measured (Fig. 2A) and found to be on average $\sim 40 \%$ shorter in the adult mutant (Fig. 2B). Note that the density of radial fibers in Neurod 1cKO is reduced compared with littermate control. Although their density was evidently lower than in controls, radial fibers were preserved in adult mutant cochlea (Fig. $2 G)$. We next measured the volume of the $\mathrm{CN}$ at the entry of the auditory nerve in coronally sectioned adult brains. The volume of the $\mathrm{CN}$ was reduced by $\sim 39 \%$ (Fig. 2C,D). Because Islet ${ }^{\text {cre }}$ is not expressed in the $\mathrm{CN}$ (Fig. $2 \mathrm{H}$ ), the size reduction is not likely because of Neurod1 deletion in the CN (Fritzsch et al., 2006) but is exclusively a secondary effect of reduced afferent input consistent with the effects of neonatal cochlear ablation previously reported (Rubel and Fritzsch, 2002). In contrast to the CN, sections of the IC showed no significant reduction in the adult mutant mice, indi- 

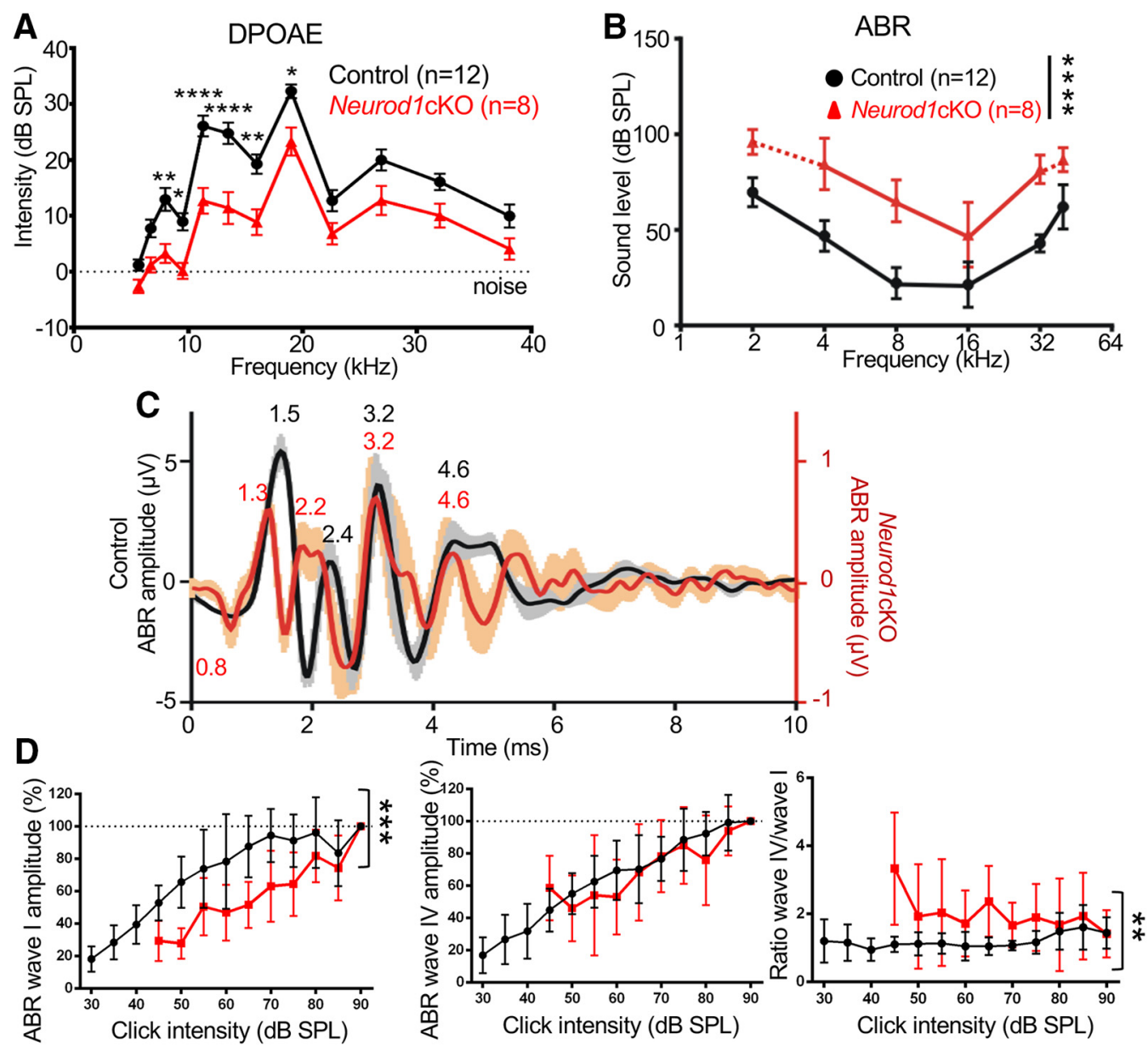

Figure 3. DPOAEs and ABRs are altered in Neurod7cKO. $A, \mathrm{OHC}$ function assessed by DPOAE shows significantly reduced levels in the low-frequency range. Data are the mean \pm SEM; $n=$ 12/control, $n=8 /$ Neurod1cK0; two-way ANOVA with Bonferroni post hoc test. ${ }^{*} p<0.05,{ }^{* *} p<0.01$, ${ }^{* * * *} p<0.0001$. B, The average ABR thresholds of control ( $n=12$ ) and Neurod7cK0 mice $(n=8)$. Five of the 8 Neurod7cKO animals did not have any response to the highest measured intensity at 2 or $40 \mathrm{kHz}$, indicated by the dotted line. Data are the mean \pm SD; two-way ANOVA. ${ }^{* * * *} p<0.0001$. C, Averaged ABR response curves evoked by an $80 \mathrm{~dB}$ SPL click; control in black, Neurod7cKO in red. The solid line shows the average and the shading indicates \pm SEM. The ABR wave amplitudes of the mutant were five times lower than the control. Averaged individual ABR wave latency values are shown by the corresponding peaks. $D$, Averaged click-evoked ABR amplitudeintensity functions normalized to the wave amplitude at $90 \mathrm{~dB}$ SPL for ABR waves I and IV, and the ratio of ABR wave IV amplitude to wave I amplitude. Data are represented as the mean \pm SD; two-way ANOVA. ${ }^{* *} p<0.01,{ }^{* * *} p<0.001$.

cating that the reduction of the $\mathrm{CN}$ does not result in a matching reduction of the IC (Fig. $2 E, F$ ).

We evaluated DPOAE, as an objective measure of the function of the cochlear OHCs and cochlear amplification, using frequency range from 4 to $38 \mathrm{kHz}$ (Fig. $3 \mathrm{~A}$ ). Based on the base-apical gradient from high to low frequencies (Müller et al., 2005), we detected significant DPOAE changes in the frequency range between 4 and $18 \mathrm{kHz}$ corresponding to the locations of the $\mathrm{OHCs}$ in the mid-apex and the apex of the cochlea. These data indicate that $\mathrm{OHC}$ dysfunction mostly correlates with the morphological disorganization of the epithelium detected in the apex (Fig. 1I-L). Hearing of mice was assessed with ABRs, which measure electrical activity associated with the propagation of acoustic information through auditory nerve fibers to higher auditory centers. The ABR thresholds of mutant mice were elevated compared with the thresholds of control animals throughout the entire measured frequency range, with a relatively even threshold shift in all frequencies averaging $\sim 35$ $\mathrm{dB}$ of SPL (Fig. 3B). Using click-evoked ABR, we evaluated waveform characteristics (Fig. 3C). ABR wave amplitudes were five times lower, but the absolute latency of peak I was shorter in Neurod1cKO mice. Wave I reflects the synchronous firing of the auditory nerve, whereas waves II-IV are attributed to the electrical activity of downstream circuits in the $\mathrm{CN}$, superior olivary complex, and IC, respectively (Martin and Rickets, 1981). We expected that CN responses should be more affected compared with IC responses because of the overall size reductions. The shorter wave I latency in Neurod1cKO indicates altered properties of SG neurons in information processing. The relative interpeak latency between peaks I and II was retained within normal limits, whereas the interval between peaks II and III was prolonged, most likely because of the reduced size of the $\mathrm{CN}$ of Neurod1cKO. The timing and distribution of ABR III and IV peaks were comparable between control and mutant mice. After normalization and direct comparison of wave I with wave IV, we found that the 3:1 ratio of controls changed to a $1: 1$ ratio in the Neurod1cKO mice (Fig. 3D). Moreover, normalization showed that although attenuated, the overall intensity function of wave IV was near normal, whereas wave I was offset even more with higher intensities. These data indicate that morphological changes in the cochlea and CN translate to ABR differences. 

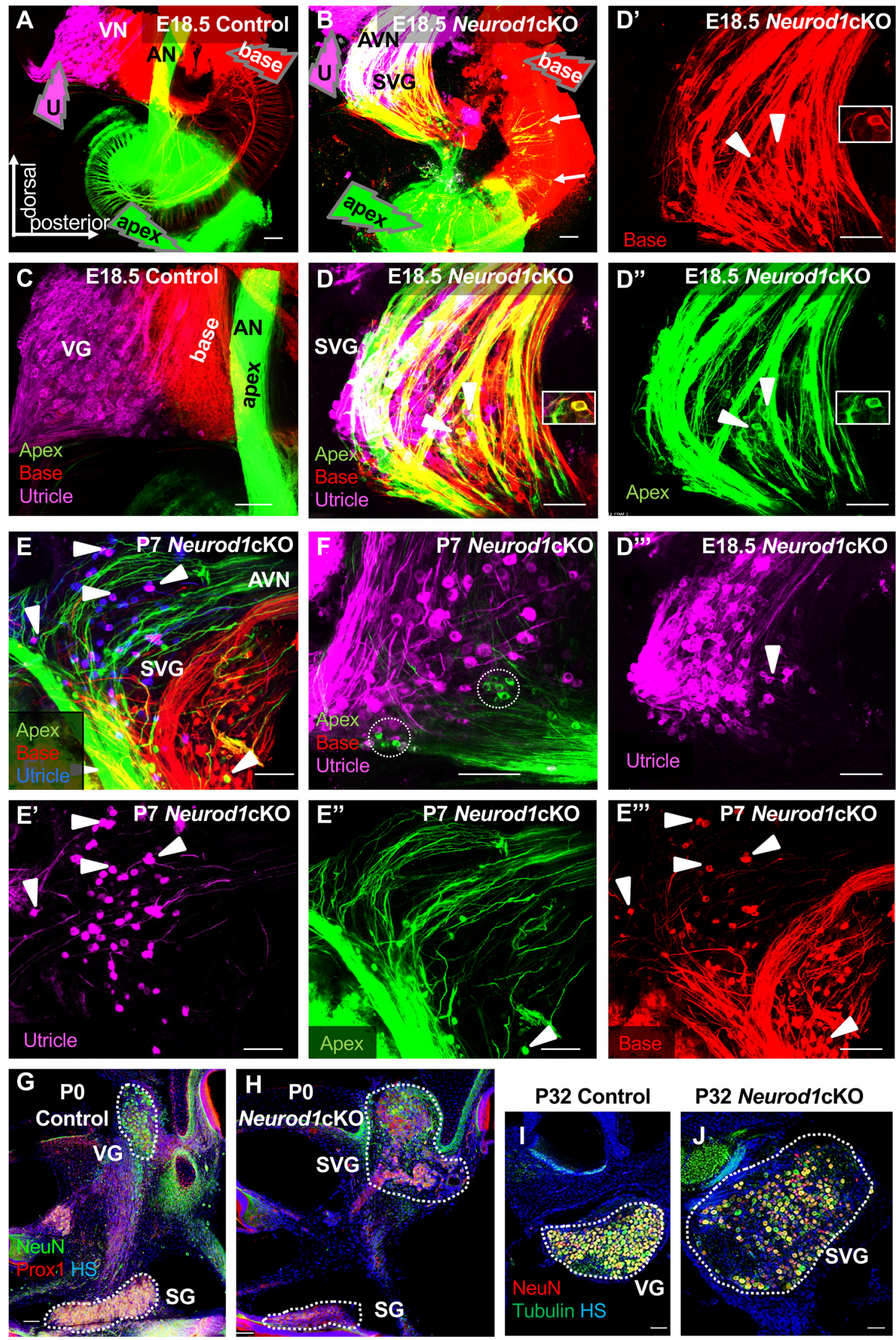

Figure 4. Translocated SG neurons form an aberrant, unsegregated spiro-vestibular ganglion in Neurod $1 \mathrm{CKO}$. $\boldsymbol{A}-\boldsymbol{F}$, Representative images of triple-dye labeling from the anterior vestibular end organs [labeled as utricle (U); magenta], apex (green), and base (red) of the cochlea shows the distribution of vestibular and SG neurons in whole-mounted collapsed stacks (A-D) and optical confocal sections $(\boldsymbol{E}, \boldsymbol{F})$. Dye injection sites are indicated by arrowheads and labeled correspondingly "apex", "base", "U" $(\boldsymbol{A}, \boldsymbol{B})$. In the control $(\boldsymbol{A}, \boldsymbol{C})$, neurons located in the vestibular ganglion (VG) of the control are only labeled by utricular dye applications $(A, C$, magenta; basal turn, red; apex, green) injections label only fibers in the AN and utricular injection labels fibers of the vestibular nerve (VN). In Neurod7cKO (B-F), neurons labeled by dyes injected into the cochlear base and apex, and the utricle combine to form an aberrant enlarged ganglion, the SVG, (Figure legend continues.) 


\section{Spiral ganglion neurons form an aberrant} "spiro-vestibular" ganglion

The application of different colored lipophilic dyes into the apex (green) and base of the cochlea (red), and anterior vestibular end organs (utricle, anterior and horizontal canal cristae; magenta) showed an aberrant distribution of spiral and vestibular ganglion neurons into a "spiro-vestibular" ganglion (SVG) complex in Neurod1cKO (Fig. $4 B, D$ ) in contrast to the vestibular ganglion of control mice with neurons exclusively labeled by anterior vestibular dye applications (Fig. 4A,C). Additionally, apex-dye application labeled fibers were detected in the base of the Neurod $1 \mathrm{cKO}$ cochlea (Fig. $4 B$, arrows). In controls, the segregated central axons of neurons labeled by dyes injected into the base and apex in the auditory nerve (AN) were separated from the vestibular nerve (labeled by the dye injection into vestibular end organs; Fig. $4 A, C)$. In contrast, the segregation of central axons was lost as basal and apical afferents completely overlapped with vestibular axons in the auditory-vestibular nerve (AVN) in Neurod1cKO (Fig. $4 B$, in detail $D, E$ ). Note that double-labeled neurons (from base/apex or base/utricular injections) were detected only in the Neurod1cKO at E18.5 and P7 (Fig. 4D-D'", $E-E^{\prime \prime \prime}$, arrowheads), indicating multiple branches of some single SG neurons and abnormal innervation within the cochlea and between the cochlea and vestibular organs. Soma size of SVG neurons labeled from dye injections into the apex of the cochlea (green) was noticeable smaller in mutants (Fig. 4F; area: $253 \pm 80 \mu \mathrm{m}^{2}$ ) compared with adjacent vestibular neurons labeled from anterior vestibular organ applications (magenta; area: $364 \pm 74 \mu \mathrm{m}^{2} ; n=80$ neurons $/ 2$ mutants, $t$ test, $p<0.001)$. We verified the tracing data using PROX1 immunocytochemistry that labels only SG neurons in wild-type (Fritzsch et al., 2010). In Neurod1cKO, PROX1 ${ }^{+}$ translocated SG neurons were detected in the area of the vestibular ganglion, thus forming a mammalian SVG (Fig. 4H) instead of the normally distinct SG in Rosenthal's canal and a vestibular ganglion between the ear and the brain of controls (Fig. $4 G$ ). The adult SVG of Neurod1cKO mice was enlarged and deformed compared with control animals (Fig. 4I,J). Our data confirm previous suggestions of such unusual migration of some SG neurons to colocalize with vestibular neurons in Pax2 $2^{\text {cre }}$ Neurod $1^{\text {loxP/loxP }}$ (Jahan et al., 2010b) or Wnt $1^{\text {cre }}$ Sox $10^{\text {loxP/loxP }}$ mice (Mao et al., 2014). We showed not only translocated SG neurons and the formation of the aberrant SVG but also single ganglion neurons having multiple

$\leftarrow$

(Figure legend continued.) as shown in whole mounts $(\boldsymbol{B}, \boldsymbol{D})$ or optical sections $(\boldsymbol{E}, \boldsymbol{F})$. In mutants, there is a tendency of fibers labeled from the apex (green) to be more anterior in the combined SVG and the combined AVN $(\boldsymbol{B})$, whereas apical fibers run in the most posterior part of the AN in control animals $(\boldsymbol{A}, \boldsymbol{C})$. Overlapping fibers (yellow) labeled by the basal and apical dye applications are present in the mutant cochlear base ( $\boldsymbol{B}$, arrows). Note that double-labeled SVG neurons can be seen after basal and apical injections ( $\boldsymbol{D}, \boldsymbol{E}$, yellow cells). The double-labeled cells after basal and anterior vestibular end organ injections can best be visualized when the dye is false colored in blue ( $\boldsymbol{E}$, double-labeled cells in magenta). $\boldsymbol{D}^{\prime}-\boldsymbol{D}^{\prime \prime}, \boldsymbol{E}^{\prime}-\boldsymbol{E}^{\prime \prime}$, Images of individual colors of the separate channels shown as a merged image in $\boldsymbol{D}$ and $\boldsymbol{E}$ (arrowheads indicate double-labeled cells from merged images). $\boldsymbol{F}$, A merged image shows only apical turn dye labeled SG neurons (green, dotted circles) near magenta labeled vestibular neurons in the SVG. Note that green cells are substantially smaller compared with the VG neurons labeled from anterior vestibular end organ dye application. Scale bars, $100 \mu \mathrm{m}$; dorsal is up and posterior is to the right in all dye-tracing images. $\mathbf{G}, \boldsymbol{H}$, Representative vibratome sections show translocated spiral ganglion PROX1 ${ }^{+}$neurons in the SVG as detected by immunohistochemistry for PROX1 (a marker for SG neurons) and NeuN (a marker for differentiated neurons) in Neurod1 1 CKO at PO. Scale bar, $100 \mu \mathrm{m}$. HS, Nuclear staining Hoechst. I, J, Immunohistochemistry for NeuN and $\beta$-tubulin in the adult control VG and Neurod1cKO SVG. Scale bar, $50 \mu \mathrm{m}$. Dotted lines indicate the boundaries of the SG, VG, and SVG. branches within the cochlea (double-labeled by base/apex injections) and between the cochlea and vestibular end organs (double-labeled by cochlear/vestibular injections) in Neurod1cKO.

\section{Spiral ganglion neurons are miswired in the cochlea of Neurod1cKO}

Having recognized the major effects of Neurod1 deletion on the cochlea and auditory nucleus size, overall physiology, and abnormal arrangement of SG neurons together with vestibular ganglion neurons in the SVG complex (Fig. 4), we next wanted to establish the details of the SG connection between the organ of Corti and vestibular organs as well as connection between the cochlea and the CN. We first investigated whether SG neuron projections are restricted to the $\mathrm{CN}$ by backfilling afferents in the ear through dye tracing injections into the $\mathrm{CN}$ (red) and cerebellum (green). These dye-tracing data showed that afferents in the cochlea of controls were strictly labeled through dye injected into the CN (Fig. 5A, red). In contrast, some SG neurons in the developing cochlea of Neurod1cKO mutants projected to the cerebellum, because their afferents were colabeled through dye injected into the cerebellum (Fig. 5B, overlapping yellow fibers in the Neurod1cKO cochlea). Note that the location of the vestibular ganglion (green dye tracing injection into the cerebellum) was anterior to the auditory nerve in controls (Fig. $5 \mathrm{~A}$ ) but posterior to the aberrant SVG ganglion in Neurod1cKO (Fig. $5 B$ ) consistent with the reorganization of anterior posterior fibers and cell distributions in the SVG (Fig. $4 B, D$ ). We next wanted to establish whether the branches of cochlear projecting neurons also reach vestibular organs in the ear by injecting dye into the cochlea, posterior canal crista and utricle ( $n=9$ at E14.5, E16.5, E18.5). Applying dye to the posterior vertical canal crista consistently labeled fibers in the cochlea in a pattern reminiscent of backfilling from the cerebellum in NeurodlcKO (Fig. 5C,D). Neurons labeled with posterior canal injections had multiple branches toward the developing organ of Corti (Fig. $5 D^{\prime}$, in detail $D^{\prime \prime}$ ) that overlapped with fibers to the base and to the posterior canal crista after dye was applied to the apex (Fig. $5 D^{\prime}$, inset, green), indicating the presence of neurons projecting to both the cochlea and the posterior canal crista. No such labeled intertwined innervation was found in the control cochlea at E14.5 nor were fibers labeled from the cerebellum (Fig. 5A). Later at E16.5, posterior vertical canal injections labeled at most a few efferent collaterals in control animals (Fig. 5E), similar to previous descriptions of branching of the efferent axons in the ear (Simmons et al., 2011; Sienknecht et al., 2014). In contrast, posterior vertical canal injection labeled SG fibers in the base, middle, and apex of the shortened cochlea of Neurod1cKO (Fig. $5 F, F^{\prime}$ ). Not only translocated SG neurons but also vestibular ganglion neurons in the SVG innervated the cochlear hair cells, as indicated by an unusual innervation pattern near inner hair cells in Neurod1cKO (Fig. $5 F^{\prime}$, arrow). These fiber terminations are comparable to rerouted vestibular fibers in neurotrophin mutants (Tessarollo et al., 2004; Fig. $5 F^{\prime}$, inset), implying that some neurons reach both the organ of Corti as well as vestibular organs.

We next investigated in more detail the branching of SG neurons within the cochlea and between the cochlea and vestibular organs. In controls, dye injections into the cochlear apex (green) and base (red) resulted in a spatially restricted labeling of SG neurons and fibers in the cochlea according to the injection site without any labeling detected from injections to anterior vestibular organs (Fig. 6A). In addition to the projection of afferents from the ear to the brain, the ear is also innervated by efferents 

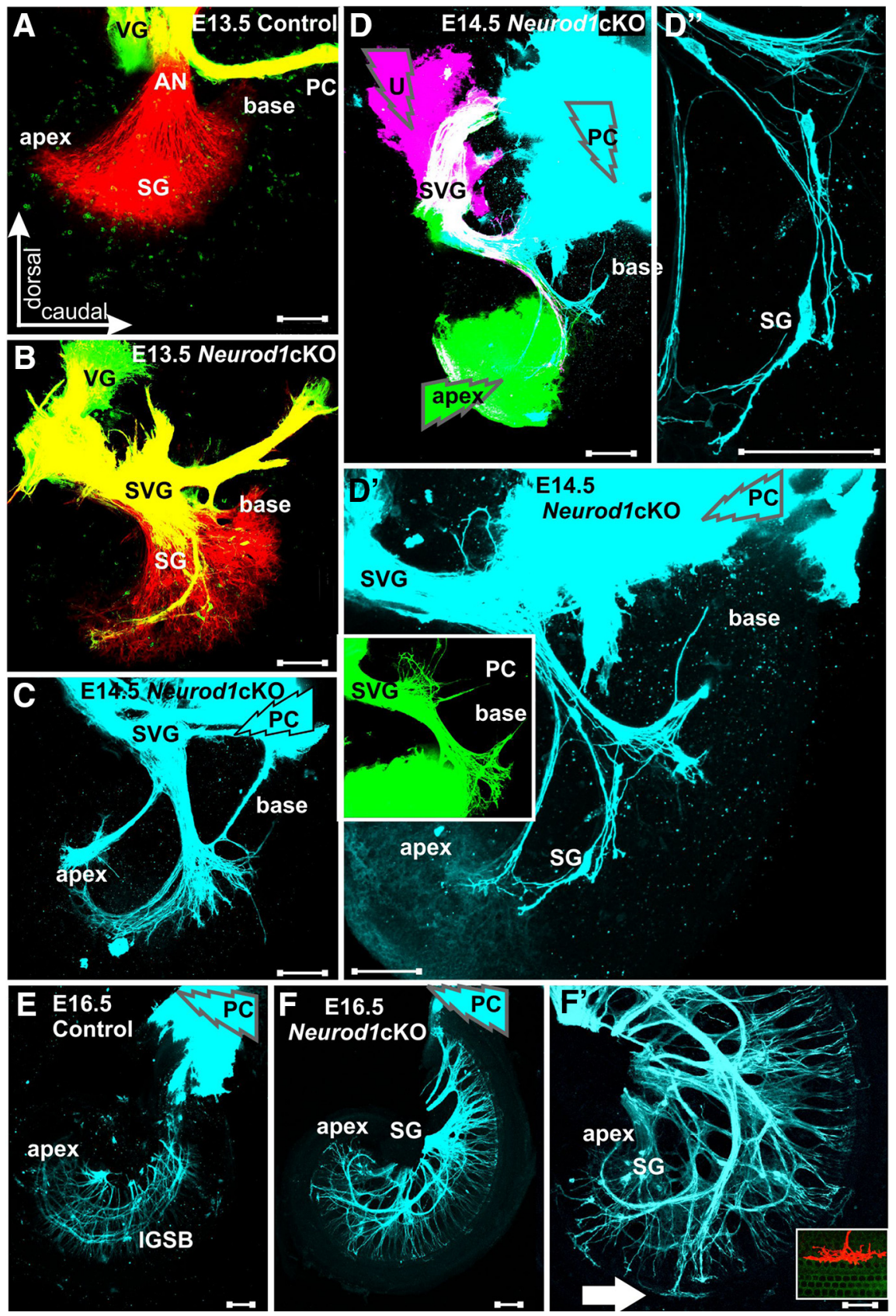

Figure 5. Loss of Neurod1 causes projection defects of SG neurons. $\boldsymbol{A}, \boldsymbol{B}, 0 \mathrm{nly}$ afferents to the cochlea are labeled by the lipophilic dye injected into the $\mathrm{CN}$ (red) but not by dye injected into the cerebellum (green) in the control at E13.5. In Neurod1 cKO littermates, some SG neurons and fibers are labeled from cerebellar injections ( $\boldsymbol{B}$, yellow, representing green overlapping with red). Note that afferents in the cochlea are distributed irregularly in mutants compared with controls $(\boldsymbol{A}$, $\boldsymbol{B}$, red). $\boldsymbol{C}-\boldsymbol{F}^{\prime}$, The cyan arrowheads show the approximate application site of the dye [posterior canal crista (PC)] that results in labeling of multiple cells and branches projecting to the developing organ of Corti in Neurod1cKO. Injection of three different colored dyes [utricle, magenta; PC, cyan; apex, green] into the E14.5 Neurod1cK0 ear shows overlap of spiral and vestibular ganglion neurons in the spiro-vestibular ganglion and magenta fibers to the cochlea $\left(\boldsymbol{C}, \boldsymbol{D}, \boldsymbol{D}^{\prime}\right)$. Highermagnification of fibers in the cochlea labeled from PC injections shows multiple neuronal branches toward the developing organ of Corti hair cells $\left(\boldsymbol{D}^{\prime \prime}\right)$. The dye injected into the apex of Neurod1cK0 ( $\boldsymbol{D}^{\prime}$, inset, green) labels fibers reaching the base as well as fibers to the posterior canal crista in a pattern reminiscent of fibers labeled from $P C$ injections. These neurons have branched axons that reach not only the brain but also the PC nerve. $\boldsymbol{E}, \boldsymbol{F}, \boldsymbol{F}^{\prime}$, After PC dye application at E16.5, only a few efferent collaterals are labeled in controls that contribute to the intraganglionic spiral bundle (IGSB). In Neurod 1 cKO at E16.5, PC dye application labels SG neurons near the apex that terminate in an unusual pattern along IHC $\left(\boldsymbol{F}^{\prime}\right.$, arrow). This pattern is comparable to the reported pattern of vestibular fibers in a replacement model of neurotrophins $\left(\boldsymbol{F}^{\prime}\right.$, inset; data from Tessarollo et al., 2004), indicating that some SG neurons project like vestibular neurons in the brain, reach vestibular end organs (such as the canal crista) and terminate at the organ of Corti in a pattern comparable to rerouted vestibular neurons. Scale bars, $100 \mu \mathrm{m}$. PC, Posterior crista; U, utricle; VG, vestibular ganglion. that are rerouted facial branchial motor neurons that end on hair cells instead of muscle fibers (Simmons et al., 2011; Fritzsch and Elliott, 2017). Typically, dye applications into the cochlea label efferent branches as the dye diffuse close to the radial fibers into the organ of Corti and efferent fibers can be distinguished by a recognizable intraganglionic spiral bundle, formed exclusively by efferents. Accordingly, in our controls, most efferent fibers were strictly either labeled by the dye applications to the apex or base in controls (Fig. $6 A^{\prime}$, red or green). Note that fibers formed evenly spaced radial bundles. Overlapping (yellow) fibers represented efferents forming the intraganglionic spiral bundle and branching to reach multiple points along the cochlea in controls (Fig. $6 A^{\prime}$ ). In contrast similar dye applications (into the base, apex, and anterior vestibular organs) labeled neurons and branches throughout the inner ear in Neurod1cKO, showing mingled bundles of fibers from the apex and base (Fig. $6 B$ ). Tracing data revealed at least three different groups of "SG neurons" within the cochlea: those restricted to base or apex, those overlapping between base and apex and those that have in addition branches to vestibular organs (Fig. $6 B-B^{\prime \prime \prime}$ ). A few SG neurons labeled from vestibular injections revealed multiple branches to reach different parts of the cochlea (Fig. $6 B^{\prime}$ ). Thus, our tracing experiments show that both the branching properties and migration properties of inner ear sensory neurons are affected by the deletion of Neurod1, generating neurons that are colocalized with vestibular neurons (Fig. 4) and reach multiple parts of the organ of Corti as well as different vestibular organs (Figs. 5, 6).

To fully evaluate efferent innervation in the cochlea, we applied lipophilic dyes into the olivocochlear bundle (green) to label efferents and into the $\mathrm{CN}$ (red) to label SG neurons and afferent radial fibers reaching the organ of Corti. In control animals, efferents formed the intraganglionic spiral bundle along SG neurons and together with afferents formed evenly spaced radial bundles (Fig. $7 A$, in detail $\left.A^{\prime}\right)$. In contrast, NeurodlcKO efferents reached outer hair cells like in control littermates but did not form the intraganglionic spiral bundle (Fig. $7 B, B^{\prime}$ ). Accordingly, the function of outer hair cells of control and Neurod1cKO was comparable to the exception of the midapex, as shown by DPOAE tests (Fig. $3 A$ ). These data support the notion that much of the intracochlear trajectory of 
efferents depends on the distribution of SG neurons but efferents can reach hair cells no matter the deviation from their normal trajectory.

Neurod1 deletion results in a loss of the tonotopic organization of the $\mathrm{CN}$

Next, we evaluated the auditory nerve fiber projections to the brainstem cochlear nucleus complex, composed of the anterior ventral $\mathrm{CN}(\mathrm{AVCN})$, posterior ventral CN (PVCN), and dorsal CN (DCN; Muniak et al., 2013, 2016). In control animals, application of different colored lipophilic dyes into the apex (green) and base of the cochlea (red), and vestibular end organs (magenta) showed the segregation of central axons of SG neurons from the base and apex in the auditory nerve and that these discrete, non-overlapping bundles were separated from the vestibular nerve (Fig. 8A,E). In contrast, the segregation of central axons of SG neurons was lost as basal and apical afferents completely overlapped in the AVN (Fig. 8B, F). Dye applications in the control cochlea showed the tonotopic organization of the $\mathrm{CN}$ subdivisions, with low-frequency-encoding fibers from the apex terminating dorsally and highfrequency-encoding fibers from the base terminating ventrally in both the $\mathrm{VCN}$ and DCN (Fig. 8A,E). In the $\mathrm{CN}$ of Neurod1cKO, fibers labeled with lipophilic dye tracing from the entire cochlea were restricted to the VCN with just a few fibers occasionally expanding to the DCN (Fig. $\left.8 B, F-F^{\prime \prime \prime}\right)$. Very large dye injections covering the basal half and apical half resulted in segregated projections with almost no space between apical and basal afferents in control animals (Fig. 8G). In contrast, comparable injections in NeurodlcKO mutants showed the entering fibers overlapped in the AVN and were mostly restricted to the VCN with just a few fibers occasionally expanding to the DCN (Fig. $8 H, I$ ). In essence, the DCN remained virtually non-innervated by cochlear afferents and the dorsal part of both AVCN and PVCN likewise received only sporadic and variable individual fibers, indicating a relocation of high-frequencyencoding basal turn fibers. In controls, cochlear afferents formed parallel fibers, the isofrequency bands (Fig. $8 C, G$, inset), whereas Neurod $1 \mathrm{cKO}$ afferents neither expanded across the entire CN nor showed a parallel fiber organization (Fig. $8 D, H, I$ ). As expected from overlapping peripheral connections of neurons in the SVG (Fig. 4) as well as neurons in cochlea reaching vestibular organs (Figs. 5, 6), we also found fibers in the $\mathrm{CN}$ of Neurod1cKO labeled by the application of lipophilic dye into vestibular end organs (Fig. $8 F^{\prime \prime}$ ). Thus, Neurod1cKO mutants have both the peripheral and central connections of spiral and vestibular neurons incompletely segregated and miswired with overlapping central axbars, $100 \mu \mathrm{m}$.
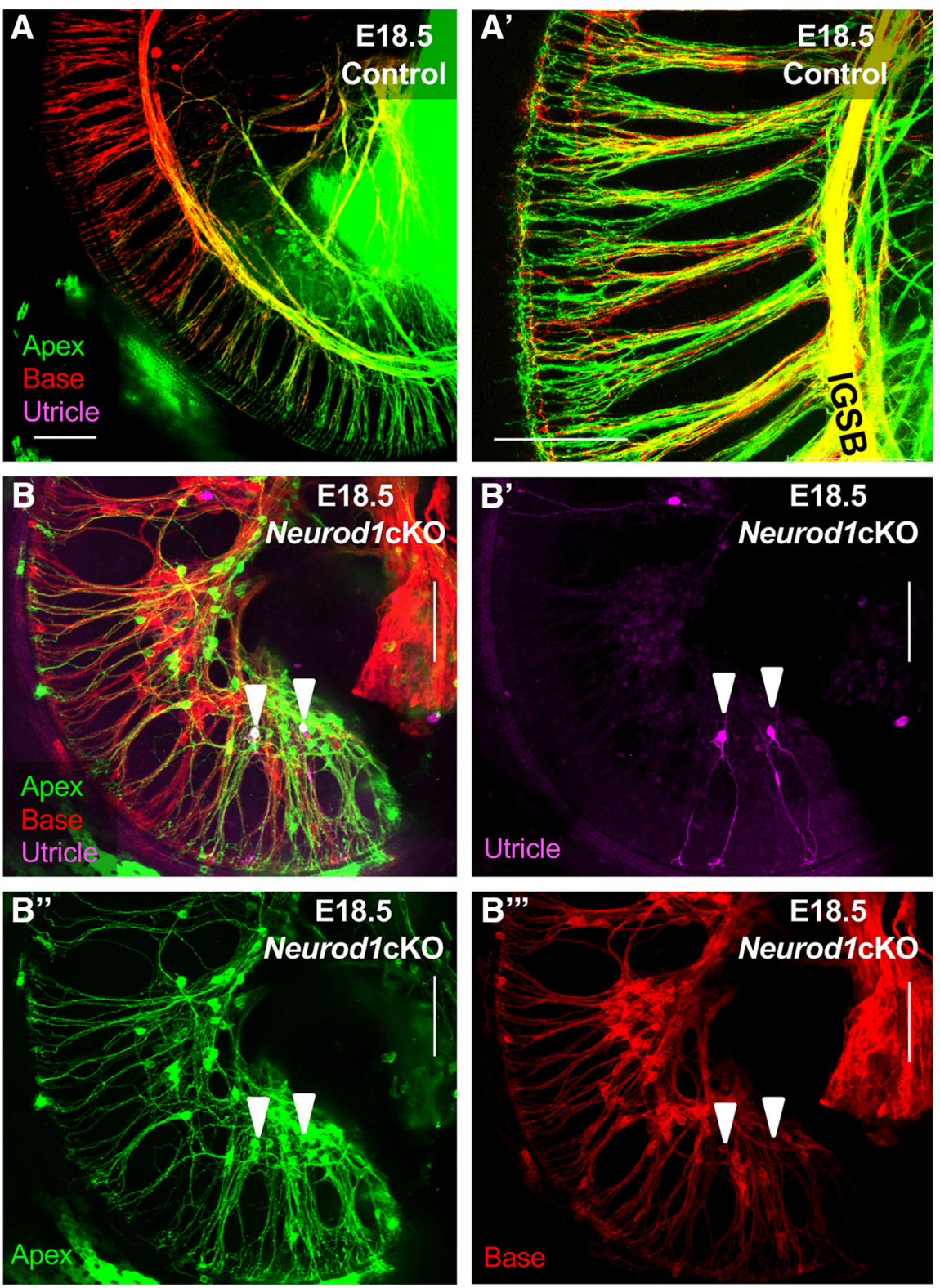

Figure 6. Distribution and multiple branches of SG neurons reveal disorganization in the Neurod1cKO cochlea. $\boldsymbol{A}, \boldsymbol{B}$, The application of different colored dyes into the apex (green), base (red), and utricle (magenta) shows a spatially restricted labeling of SG neurons and fibers based on the injection site in controls $(\boldsymbol{A})$, whereas both fibers and neurons are labeled throughout the Neurod1cK0 cochlea by the utricle, base and apex injections $(\boldsymbol{B}) . \boldsymbol{A}^{\prime}$, In controls, overlapping (yellow) efferent fibers superimpose in the intraganglionic bundles (IGSB). Some efferent fibers are labeled either by the dye application to the apex or base in controls (red or green) and together with afferents form evenly spaced radial bundles. $\boldsymbol{B}^{\prime}-\boldsymbol{B}^{\prime \prime \prime}$, images of individual colors of the separate channels shown as a merged image in $\boldsymbol{B}$. Note that $S G$ neurons near the apex are double labeled with magenta (utricle) and green (apex) as indicated by arrowheads, and show in addition two or more local branches to different parts of the cochlea $\left(\boldsymbol{B}^{\prime}\right)$. Scale

ons of SG neurons in the auditory-vestibular nerve and limited projections of widely ramifying fibers to the ventral part of $\mathrm{CN}$ that showed variable and inconsistent segregation (Fig. 8J, summary).

Since the size of the CN depends on the full complement of afferents and the survival of CN neurons depends on innervation (Rubel and Fritzsch, 2002; Syka, 2002), we next investigated the survival of one class of neurons that receive input from auditory nerve fibers through the large end bulbs of Held, the bushy cells in the AVCN (Muniak et al., 2016). Although the size of the AVCN in Neurod1cKO was reduced, SG afferents of Neurod1cKO and 

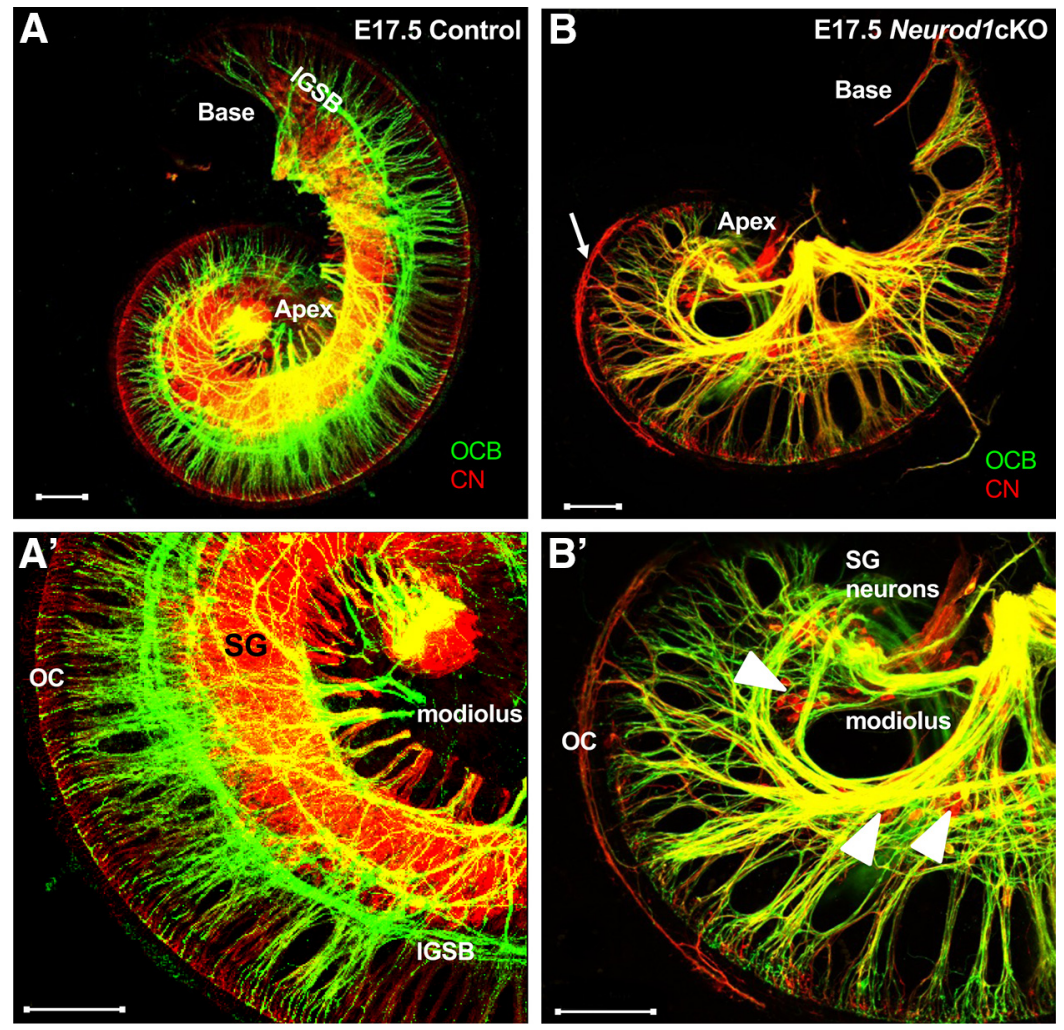

Figure 7. Disorganized inner ear efferents reach the organ of Corti in the Neurod7cK0 cochlea. $A, A^{\prime}$, In control animals, the injections of lipophilic dye into the olivocochlear bundle $(O C B$; green) labels inner ear efferent fibers forming the intraganglionic bundle (IGSB) and dye application into the $\mathrm{CN}$ (red) labels SG neurons and afferent radial fibers reaching the organ of $\mathrm{Corti}(\mathrm{OC})$. $\boldsymbol{B}$ $\boldsymbol{B}^{\prime}$, In Neurod1cK0, the same dye applications label the scattered SG neurons (red dye-labeled; arrowheads) in the cochlea and show strong efferent innervation that lacks an IGSB formation. Note also the profound innervation of the $\mathrm{OHC}$ region of the $\mathrm{OC}$ only in the apex of the Neurod1cK0 mutant ( $\boldsymbol{B}$, arrow). Scale bars, $100 \mu \mathrm{m}$.

control formed morphologically comparable clusters of boutons that wrap the somas of their targets with the end bulbs of Held, as shown by excitatory VGLUT1 synaptic marker labeling (Fig. 9A$\left.B^{\prime}\right)$. However, innervation patterns of afferents outside the overlapping area in AVCN of NeurodlcKO (Fig. 8D, H,I) suggest an unusual distribution that does not conform to the regular pattern of stratified afferent projections in controls (the isofrequency bands in Fig. 8C,G, inset), which represent the tonotopic input of the organ of Corti onto the CN (Muniak et al., 2013, 2016).

\section{c-Fos shows a distorted frequency map representation in the $\mathrm{CN}$}

We next wanted to investigate whether SG afferents formed functional synapses onto AVCN bushy cells and how distinct frequencies are mapped throughout the system in terms of the activation of the immediate early response of c-Fos, a technique shown to allow such mapping (Tomková et al., 2015; Karmakar et al., 2017). We subjected freely moving adult mice (2 months old) to pure tone auditory stimulation with $15 \mathrm{kHz}$ pips at $75 \mathrm{~dB}$ for 90 min in a sound-proof chamber. We used a $15 \mathrm{kHz}$ tone that drives cochlear activation near the middle in controls and likely also in NeurodlcKO mutants, assuming the apex and base shorten equally. Consistent with previous reports (Karmakar et al., 2017), in control mice, we found a narrow band of $\mathrm{c}-\mathrm{Fos}^{+}$cells near the center of the AVCN, corresponding with the known isofrequency representation (Fig. 9C). In contrast, in NeurodlcKO, $15 \mathrm{kHz}-$ stimulation activated AVCN neurons but the c-Fos activation area was spread all over the AVCN and the number of activated neurons was doubled (Neurod1cKO $20.7 \pm$ 2.5; control $9.8 \pm 1.4, p=0.0099$; Fig. $9 D)$. These data indicate that the pure tone stimulation of $15 \mathrm{kHz}$ activated AVCN neurons but the tonotopic precision of SG axon targeting was degraded with a spread of activation to many surrounding frequencies.

Frequency tuning, intensity, and temporal coding properties of IC units are distorted in Neurod $1 \mathrm{cKO}$

With this background on neuroanatomical, quantitative and frequency related c-Fos activity changes in mind, we next investigated the tuning properties of IC neurons at various frequencies as well as other parameters of their function i.e., two-tone suppression, intensity, and temporal resolution as the IC is the first level of auditory space map projection and shows some amelioration of primary afferent dysfunction (Buran et al., 2010; Pelgrim et al., 2018).

The study of the tuning characteristics of IC neurons revealed striking differences in the shape of the excitatory receptive fields between the Neurod1cKO and control mice. Instead of simple narrow V-shape receptive fields (a mono-peak response) seen in the controls, we recorded mostly wide receptive fields with two or more peaks in clusters of IC neurons of Neurod1cKO mice (Fig. 10A), suggesting multiple inputs from the lower levels of the auditory system. Two-tone stimulation, used to detect inhibitory areas surrounding the excitatory tuning curves, showed the presence of low- and high-frequency sideband inhibitory areas in controls, and small and disorganized inhibitory areas in NeurodlcKO (Fig. 10B). Because we mostly recorded the multiple-unit activity of neuronal clusters in the IC, we wanted to establish whether the multipeaked broad tuning curves in mutants are formed by the integration of responses of several neurons with different best frequencies or whether they reflect a miswiring of auditory fibers occurring below the IC. We found that the multipeaked broad tuning curves in the responses of neuronal clusters were also observed in the tuning curves of isolated single units recorded at the same electrode (Fig. 10C-H).

The investigation of responsiveness of IC units of the mutant mice to different sound frequencies revealed a frequency range with a limited presence of CFs from 9 up to $28 \mathrm{kHz}$ (Fig. 11A). These features are consistent with the observed shortening of the cochlea, and the reduced amplitude of the ABR. In addition, the excitatory thresholds of IC units were higher in mutants than in control animals in all measured frequencies (Fig. 11A). One commonly used metric of auditory tuning is the "quality factor", or Q, defined as the CF divided by the bandwidth, typically measured 10 $\mathrm{dB}$ above the minimum threshold. Comparing the data of control and mutant mice, the tuning curves recorded at individual electrodes in the IC of mutant mice had a significantly lower quality factor $\mathrm{Q}_{10}$, indicating that their frequency selectivity was worse (Fig. 11B).

To assess the responsiveness of neurons to sound intensity, we recorded the responses of IC multiunits to $\mathrm{WN}$ bursts of variable 

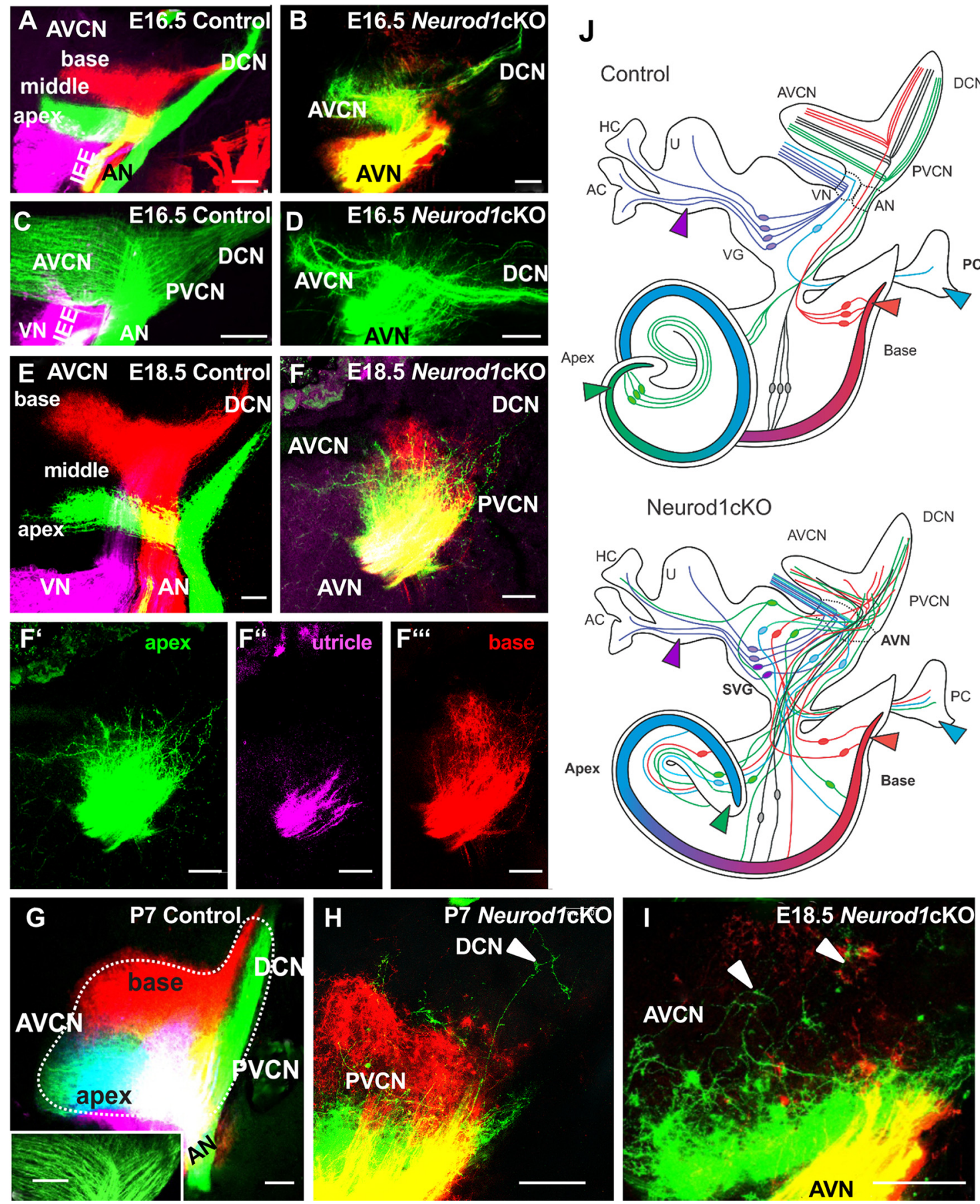

Figure 8. Neurod1cKOSG neurons project unsegregated widely ramifying central axons to the CN. $\boldsymbol{A}, \boldsymbol{C}, \boldsymbol{E}$, Injections of different colored dyes (magenta, vestibular organs; red, base; green, apex) label distinct bundles of neuronal fibers (AN) projecting to the CN and vestibular nuclei in controls. The tonotopic organization of the CN subdivisions in controls is shown in the AVCN and the DCN with low-frequency fibers labeled from the apex and high-frequency from the base and no labeling between (the middle cochlea; $\boldsymbol{A}, \boldsymbol{E}, \boldsymbol{G}$ ). The only mixed bundle (yellow) are inner ear efferents (IEEs; A). In Neurod1cK0, neuronal fibers completely overlap forming a mixed-labeled AVN (yellow) and are restricted to the ventral part of the CN with just a few fibers occasionally expanding to the DCN $(\boldsymbol{B}, \boldsymbol{D}, \boldsymbol{F})$. The overlapping fibers in the AVCN indicate a loss of the tonotopic organization of the central projections $(\boldsymbol{B}$, yellow fibers). $\boldsymbol{C}, \boldsymbol{D}$, Higher-magnification shows central afferents as parallel fibers (isofrequency bands) in control AVCN and DCN, whereas central projections of afferents in mutants branch in a restricted and erratic pattern in the $C^{-N}$ (green dye injection into the apex). $\boldsymbol{E}$, $\boldsymbol{F}$, The tonotopic organization of the CN subdivisions with low-frequency-encoding fibers from the apex (green) terminating ventrally and high-frequency-encoding fibers from the base (red) terminating dorsally in the control AVCN and DCN. In contrast, disorganized fibers labeled by dye injected in the apex of Neurod1cKO project into the ventral part of the AVCN, whereas virtually only sporadic fibers labeled by basal dye injection reach only PVCN subdivisions. $\boldsymbol{F}^{\prime}-\boldsymbol{F}^{\prime \prime}$, , Single color images show the incomplete cochlear projection segregation as well as some "vestibular" fibers projecting to the $\mathrm{CN}$ of Neurod1cKO $\left(\boldsymbol{F}^{\prime \prime}\right)$ that never happens in control animals $(\boldsymbol{A}, \boldsymbol{C}, \boldsymbol{E})$. $\boldsymbol{G}$, Very large lipophilic dye injections result in barely segregated projection with almost no space between apical and basal afferents in controls. Inset, Cochlear afferents organized as parallel fibers in isofrequency bands. $\boldsymbol{H}, \boldsymbol{I}$, Comparable large dye injections label sporadic and divergent fibers mostly in the ventral part of the CN of Neurod1CKO at P7 and E18.5. Note that single afferents project widely ramifying across the CN complex ( $\boldsymbol{H}, \boldsymbol{I}$, arrowheads). Scale bars, $100 \boldsymbol{\mu m}$. J, Schematics of changes in Neurod 1CKO: (1) SG neurons are miswired and misplaced into an aberrant SVG, (2) overlapped central auditory and vestibular afferents form the AVN, and 3) the CN has reduced projections without tonotopic organization both in terms of branch distribution within the cochlea and to the CN. The triangles indicate the application sites of the dyes in the base, apex, utricle (U), and posterior canal crista (PC). AC, Anterior crista; HC, horizontal crista; VG, vestibular ganglion; VN, vestibular nerve. 

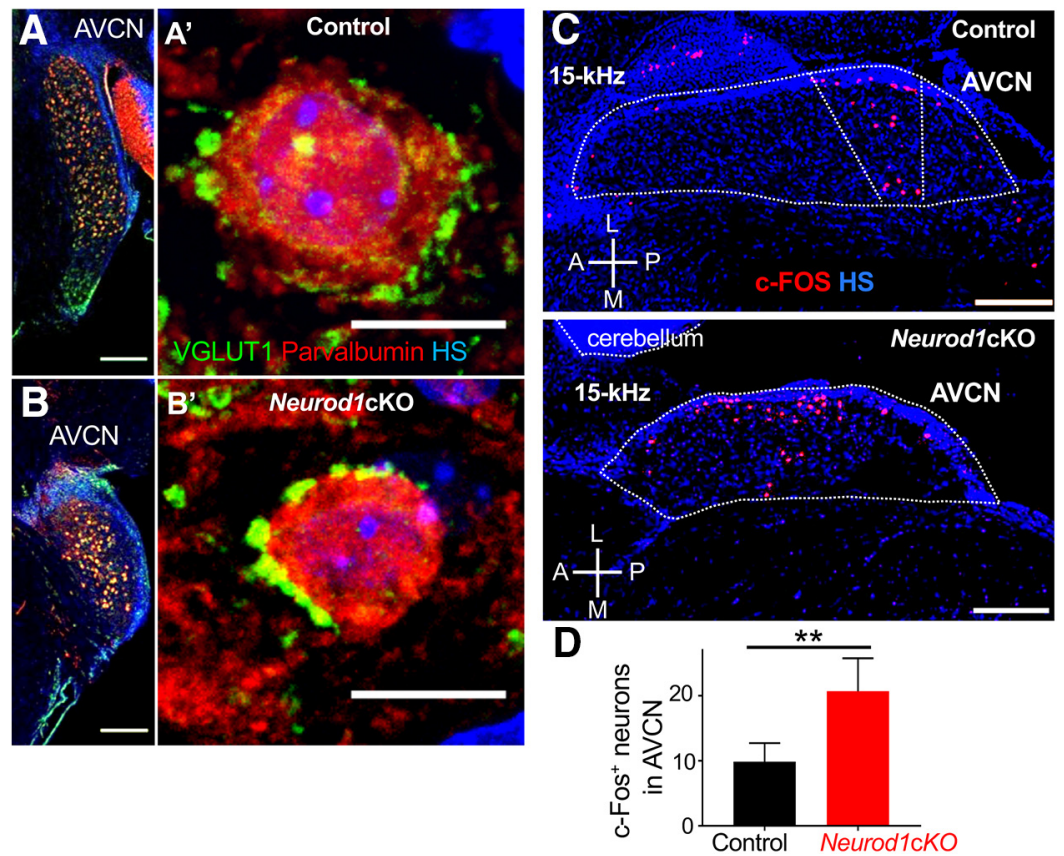

Figure 9. The formation of isofrequency bands in the anteroventral CN of Neurod1cK0 is altered. $\boldsymbol{A}, \boldsymbol{A}^{\prime}, \boldsymbol{B}, \boldsymbol{B}^{\prime}$, Auditory nerve synaptic terminals of control and mutant mice are comparable, as anti-VGLUT1 labels the endbulb of Held synapses and antiparvalbumin labels the bushy cell soma in the AVCN of the adult control and Neurod1cKO. C, Activated neurons are labeled by anti-c-Fos antibody in response to $15 \mathrm{kHz}$ pure tone stimulation in the AVCN of 2 months old controls and Neurod $1 \mathrm{cKO}$. Dotted line indicates boundaries of the AVCN. A, Anterior; L, lateral; M, medial; P, posterior. $\boldsymbol{D}$, The total number of c-Fos ${ }^{+}$neurons determined in the sections of the AVCN of control $(n=4)$ and Neurod1cKO mice $(n=4)$. Two sections were evaluated per each individual mouse. Values are mean \pm SEM. ${ }^{* *} p=0.0099, t$ test. Scale bars: $200 \mu \mathrm{m} ; \boldsymbol{A}^{\prime}, \boldsymbol{B}^{\prime}, 10 \mu \mathrm{m}$. HS, Nuclear staining Hoechst.

intensity and used them to construct RIFs. The percentage of multiunits with non-monotonic responses (i.e., encoding the increasing intensity initially by increasing spike rate, and then decreasing spike rate as sound intensity is further increased) was doubled in the IC of Neurod $1 \mathrm{cKO}(35 \%)$ compared with controls $(16 \% ; p<0.0001 ;$ Neurod $1 \mathrm{cKO} n=9 ; 432$ IC recording sites and control $n=10 ; 480$ IC recording sites; Fig. 11C). Saturated rate intensity functions were present only in $7 \%$ of the measured IC multiunits in Neurod1cKO compared with $37 \%$ in the controls $(p<0.0001)$, whereas monotonic rate-intensity functions represented $55 \%$ of the recorded neuronal clusters in NeurodlcKO compared with $47 \%$ in the controls $(p=0.0007)$. As a result, the IC multiunits in NeurodlcKO had a narrower dynamic range $(23.38 \pm 0.31 \mathrm{~dB}$ vs control $24.23 \pm 0.29 \mathrm{~dB}, p<0.05)$ as well as significantly lower maximum response magnitudes (NeurodlcKO $16.96 \pm 0.44$ spikes/s; control $25.26 \pm 0.51$ spikes/s, $p<0.0001$; Fig. 11D). Additionally, it was necessary to use significantly higher WN intensity in Neurod $1 \mathrm{cKO}$ to reach the R10, representing $10 \%$ of the RIF response magnitude (53.8 \pm $0.5 \mathrm{~dB}$ SPL; control $34.3 \pm 0.4 \mathrm{~dB}$ SPL, $p<0.0001$; Fig. $11 D$ ), as a consequence of a higher excitatory threshold. However, at this low suprathreshold noise level, Neurod1cKO IC units showed facilitated evoked activity $(0.15 \pm 0.0029$ vs control $0.12 \pm$ $0.00091, p<0.0001)$. We also observed significantly higher spontaneous activity in the NeurodlcKO IC units than in controls (Neurod1cKO $6.21 \pm 0.34$ spikes/s; control $1.5 \pm 0.11$ spikes/s, $p<0.0001$; Fig. 11D).

We performed acoustical stimulation of the IC units with two types of click trains, a long one with changing frequency of clicks in the train and a train of five clicks with different interclick intervals from 100 up to $5 \mathrm{~ms}$. In Neurod $1 \mathrm{cKO}$, this revealed a significantly lower efficiency in recognizing individual clicks in the complex train (Neurod1cKO $23.96 \pm$ $1.07 \%$; control $49.86 \pm 1.47 \%, p<$ 0.0001 ; Fig. 11E); and a lower degree of response synchronization with clicks in the train for the whole range of interclick intervals, suggesting a reduction in the precise response timing in the NeurodlcKO (Fig. 11F).

\section{Auditory behavior of Neurod $1 \mathrm{cKO}$ is altered}

Previous work has demonstrated that even minor frequency presentation mistakes in the CN can lead to system effects, revealed by altered auditory behavior (Karmakar et al., 2017; Cruces-Solís et al., 2018). The thresholds and growth of the ASR were measured for tonal and $\mathrm{WN}$ startle stimuli in a continuous-noise and in a noiseless background. In mutants, ASR thresholds were significantly reduced for startle tone stimuli of $8 \mathrm{kHz}$ (Neurod1cKO $72.14 \pm 2.67$ dB SPL; control $81.43 \pm 3.78 \mathrm{~dB}$ SPL, $p<0.001 ; n=$ 8 /group) and $16 \mathrm{kHz}$ (Neurod $1 \mathrm{cKO}$ $66.43 \pm 6.27 \mathrm{~dB}$ SPL; control $83.57 \pm 3.78$ $\mathrm{dB}$ SPL, $p<0.0001$ ), suggesting that instead of perceiving a pure tone, mutants perceive noise-like stimuli. This is further supported by the fact that the startle thresholds of mutants in response to pure tone stimulation were similar to those for WN (Fig. 12A). The differences in thresholds in response to $\mathrm{WN}$ between control and mutant mice were not significant. Consistent with the lower dynamic range, lower maximum response and higher percentage of non-monotonic responses of IC multiunits (Fig. 11C,D), we found that Neurod1cKO mice had a reduced amplitude of the startle response at higher intensities of noise startle stimuli (Fig. $12 \mathrm{~B})$. However, the $16 \mathrm{kHz}$ stimulation resulted in significantly larger amplitudes of the response at low and middle intensities in mutants (Fig. 12C). To further assess complex auditory discrimination behavior in Neurod1cKO mutants, we subjected control and mutant adult mice to a PPI paradigm, which is an operational measure of sensory-motor gating. We used either WN or pure tone pips at increasing intensities as a non-startling acoustic stimulus (prepulse) that preceded the startle stimulus in a quiet background. PPI with WN as the prepulse resulted in mutants with a larger inhibition of the startle response than in controls (Fig. 12D). PPI growth functions demonstrated that the prepulse WN bursts can significantly modulate the startle response at sound pressures as low as $10 \mathrm{~dB}$ SPL in mutant mice, indicating heightened sensitivity of the central auditory system. Similarly, subsequent comparisons revealed that the PPI of Neurod1cKO for 16 $\mathrm{kHz}$ prepulses (a preserved audible frequency in the mutant IC) was enhanced starting at $40 \mathrm{~dB}$ SPL compared with control mice (Fig. 12E). Additionally, mutant mice showed higher sensitivity to increasing intensities of the continuous background $\mathrm{WN}$ than controls (Fig. 12F). Thus, NeurodlcKO displayed hyperacusislike behavioral ASR and PPI responses parallel to animal models with noise-induced cochlear neuropathy, which is also associated with increased behavioral salience of still-audible sounds (Hickox and Liberman, 2014). 


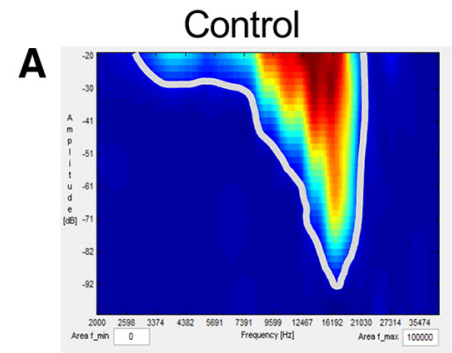

Neurod1cKO 2

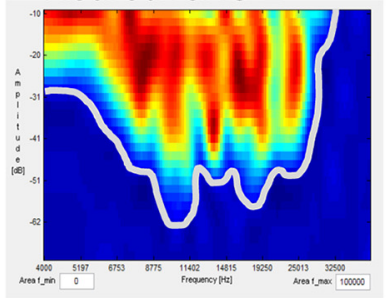

C

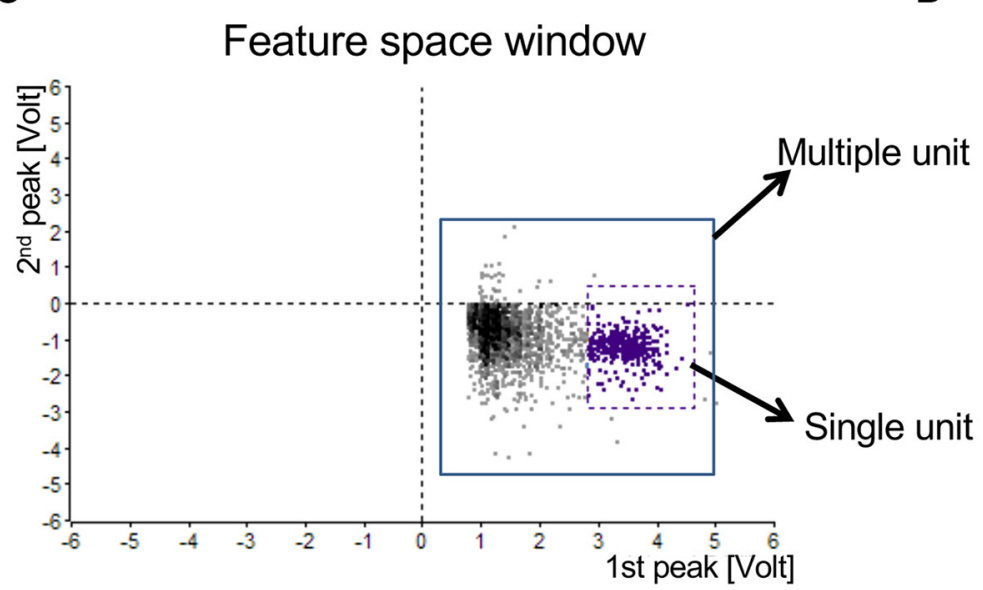

Neurod1cKO 1

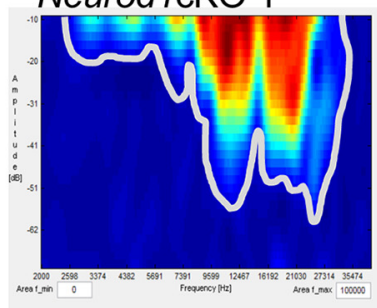

Neurod1cKO 3

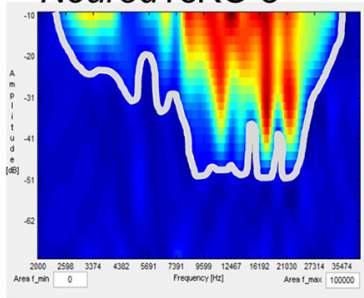

1st peak [Volt]
Neurod1cKO \#3
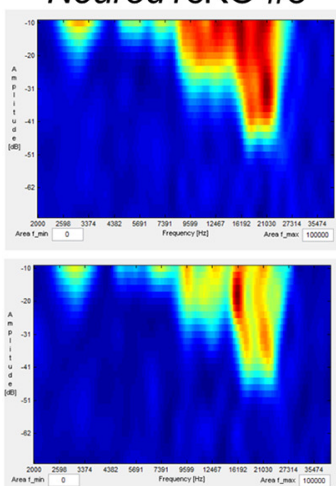

$\mathbf{F}$

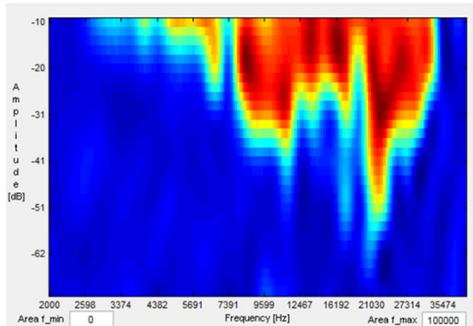

Neurod1cKO \#4
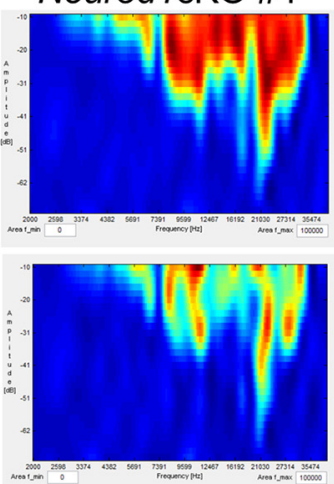

H
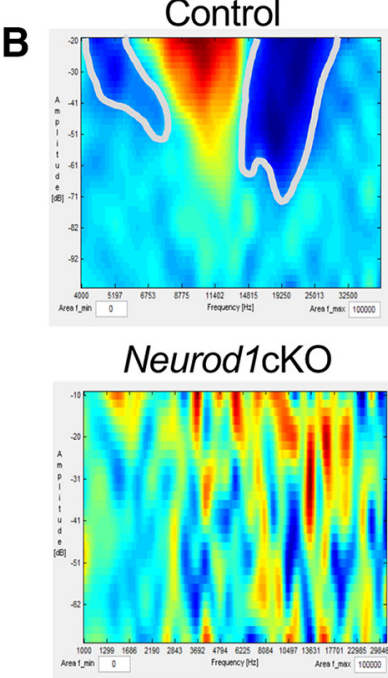

Receptive fields

Neurod1cKO \#1
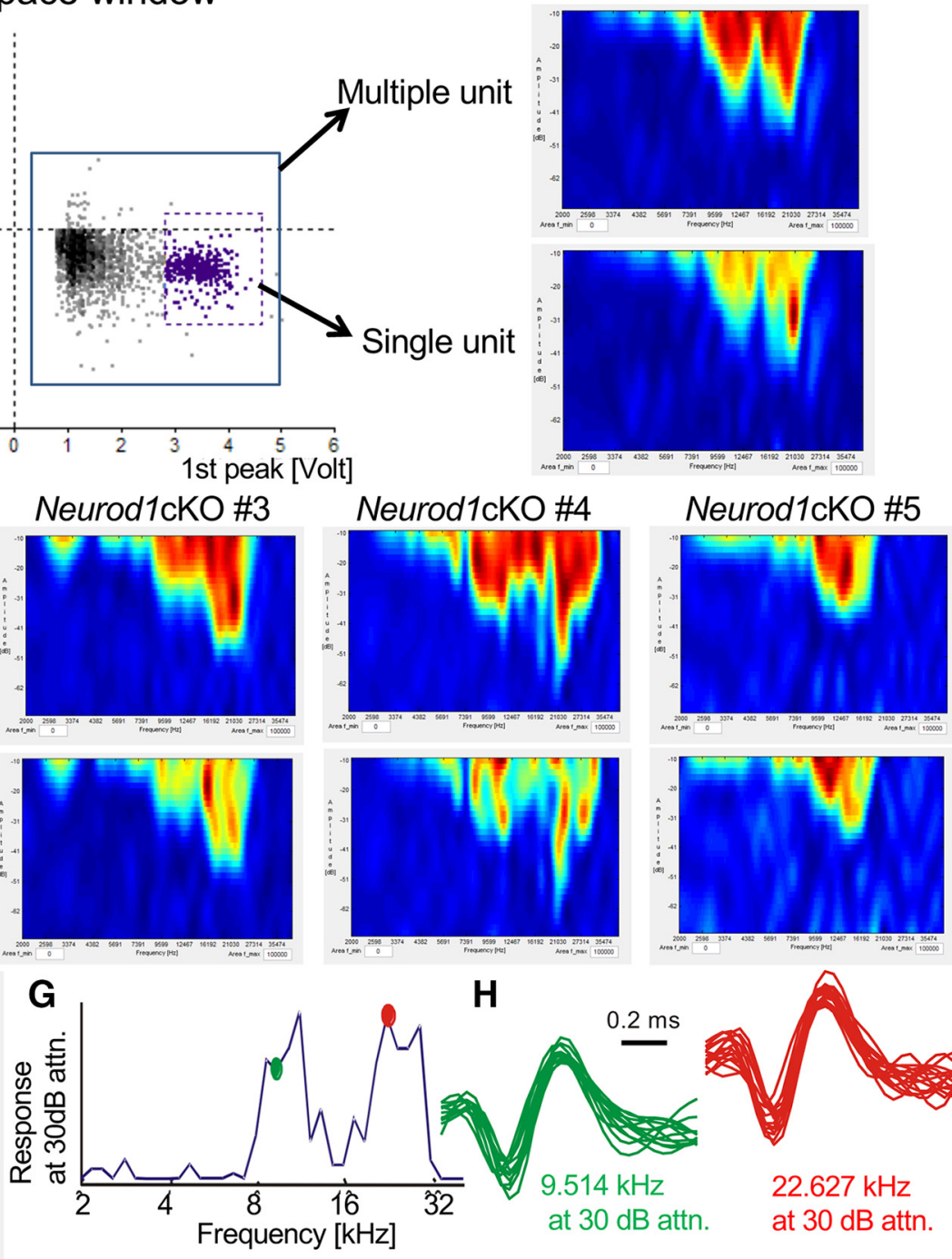

Figure 10. Deletion of Neurod1 affects the IC tuning curves of multiple and isolated single units. $\boldsymbol{A}$, Representative examples of tuning curves recorded in the IC in control and Neurod 1 cKO mice. $\boldsymbol{B}$, Response map to two-tone stimulation (fixed tone of $13 \mathrm{kHz}$ ), shows low- and high-frequency sideband inhibitory areas in control (the areas are outlined by white lines) and small and disorganized inhibitory areas in Neurod1cK0 mice. C, Feature space window (in which each action potential is represented by a single point) with a delineated cluster of action potentials (purple dashed box) belonging to isolated single units recorded at the electrode channel. Action potentials corresponding to multiple unit activity are within the larger box (Figure legend continues.) 

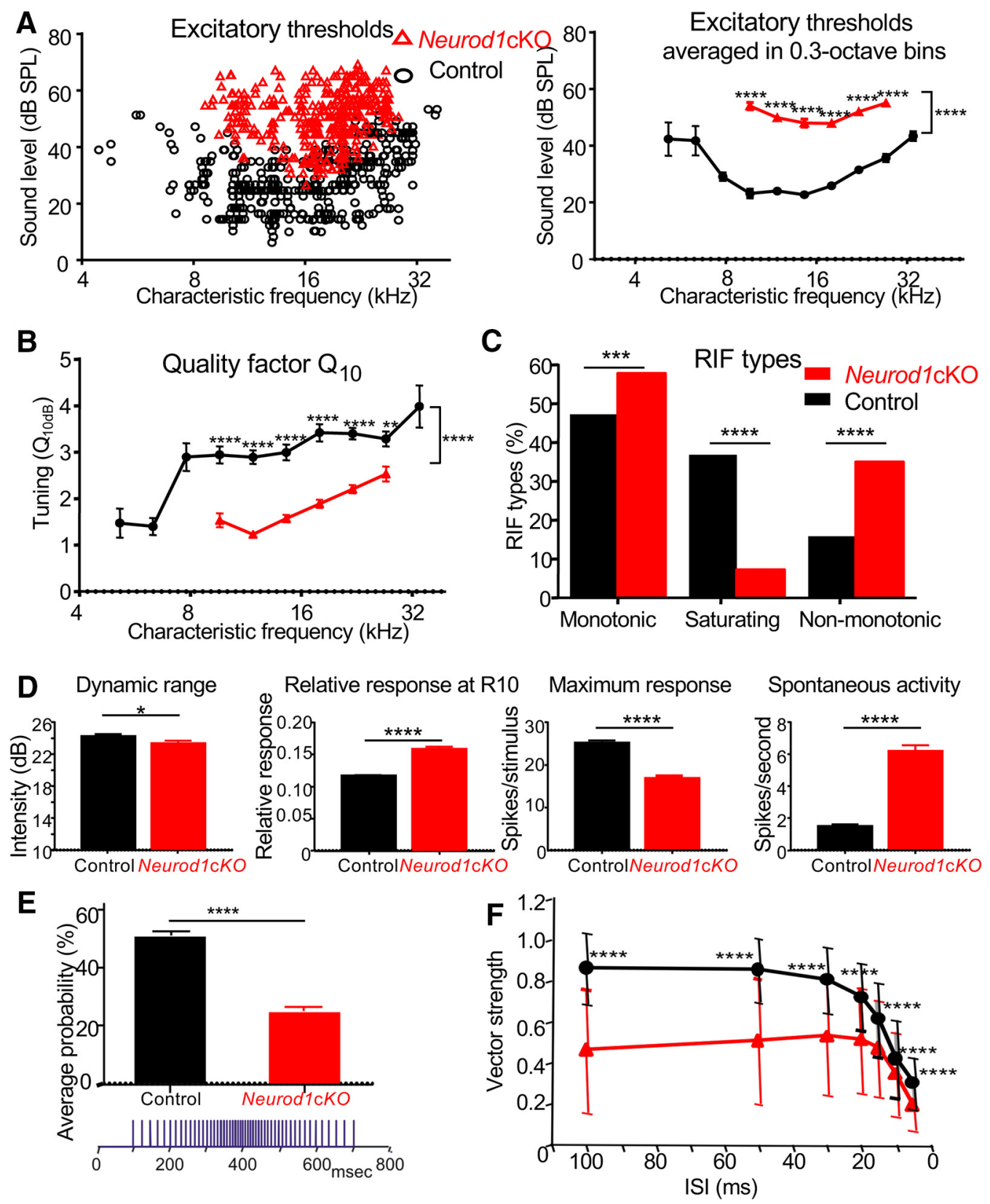

Figure 11. Characteristics of IC neurons are affected by acoustic information processing in the periphery. $A$, The dependence of $\mathrm{IC}$ excitatory thresholds on the $(\mathrm{C}$ of neuronal clusters (multiunits) in control and Neurod1cKO mice. Excitatory thresholds are shown as individual units and as averages in 0.3 octave bins. Data are mean \pm SEM. Two-way ANOVA with Bonferroni post hoc test. ${ }^{* * * *} p<0.0001$. $B$, Sharpness of the neuronal tuning expressed by quality factor $\mathrm{Q}_{10}$ (the ratio between the $C F$ and bandwidth at $10 \mathrm{~dB}$ above the minimum threshold) averaged in 0.3 octave bins. Data are represented as the mean \pm SEM; two-way ANOVA with Bonferroni post hoc test. ${ }^{* * *} p<0.0001,{ }^{* *} p<0.01$. C, Percentages of strictly monotonic, saturating and non-monotonic RIFs. Fisher's exact test. ${ }^{* * *} p<0.001,{ }^{* * * *} p<0.0001$. D, Comparison of the RIF parameters in control and Neurod1cK0 mice: dynamic range, relative response at the RIFs point R10 (10\% of the RIF response magnitude), maximum response magnitude and spontaneous activity. Data are the mean $\pm S D$; unpaired $t$ test. ${ }^{*} p<0.05,{ }^{* * * *} p<0.0001$. For all extracellular recordings Neurod 1 cK0 ( $n=9 ; 432$ units from the IC) and control mice ( $n=10 ; 480$ units) were used. $E$, The average probability of a response to individual clicks in the series of clicks. Inset, Diagram of the clicks train, where the rate continuously speeds up and then slows down again (mean $\pm \mathrm{SEM} ; n=10 /$ control, $n=9 / \mathrm{Neurod}_{1} \mathrm{KKO}$; ${ }^{* * *} p<0.0001$, unpaired $t$ test). $\boldsymbol{F}$, Degree of synchronization for different

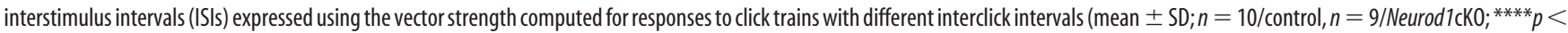
0.0001, two-way ANOVA with Bonferroni post hoc test).

\section{$\leftarrow$}

(Figure legend continued.) (indicated by solid blue line). $\boldsymbol{D}, \boldsymbol{E}$, Examples of the tuning curves representing multiple unit activity and the activity of a single isolated unit from five different recordings in Neurod 1 cKO mice. F, Tuning curve of a multiple unit in Neurod7cKO mice; for the same recording, frequency profile of the same response to tonal stimulation at different frequencies with bimodal frequency tuning (at $30 \mathrm{~dB}$ sound attenuation) is calculated from singleunit activity $(\boldsymbol{G})$. $\boldsymbol{H}$, Action potential curves, recorded at two different points marked with the corresponding color in the frequency profile at $(\boldsymbol{E})$, demonstrating very similar spike shapes and therefore classified as generated by the same neuron (single unit).
Neurod 1 deletion results in aberrant vestibular function

Finally, we investigated the possibility of interference of vestibular function of Neurod $1 \mathrm{cKO}$ with auditory stimuli. This would be expected given the distribution of SG neurons in the aberrant SVG, shared central projections after labeling vestibular and cochlear afferents, and labeled neurons in the SG by dye injected into vestibular organs (Figs. 4, 6, 8). Thus, these results demonstrate interconnections of afferents between cochlear and vestib- 

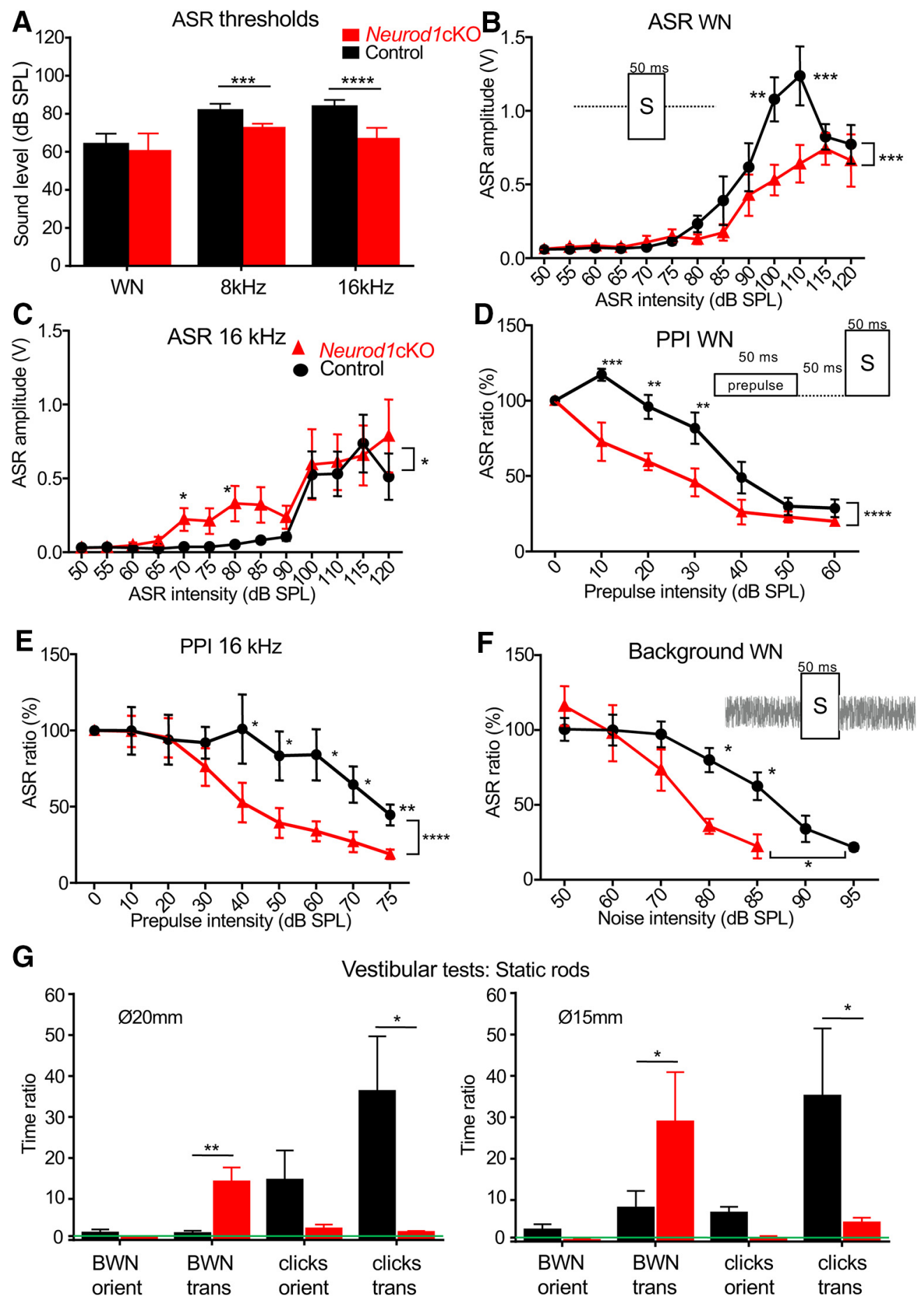

Figure 12. ASR and PPI responses are altered in Neurod $1 \mathrm{CKO}$. A, Thresholds of ASRs for WN, and tone pips at 8 and $16 \mathrm{kHz}$ in control and Neurod $7 \mathrm{CKO}$ mice. Data are the mean \pm SEM, $n=8 / \mathrm{group}$. Statistical significance determined by the Holm-Sidak method, $t$ test. ${ }^{*} p<0.05,{ }^{* *} p<0.01,{ }^{* * *} p<0.001$. Amplitude-intensity ASR functions for WN (B), and for tone pips of $16 \mathrm{kHz}(\boldsymbol{C})$ in control and Neurod 1 cKO mice. Data are the mean \pm SEM, $n=8$ /group. $\boldsymbol{D}$, Efficacy of the WN and $(\boldsymbol{E}) 16 \mathrm{kHz}$ tone prepulse intensity on the relative ASR amplitude; $100 \%$ corresponds to the ASR amplitude without a prepulse (uninhibited ASR). Data are the mean \pm SEM, $n=8$ /group. $F$, Impact of a continuous background WN with increasing intensity on the relative ASR amplitude; $100 \%$ corresponds to the amplitude of uninhibited ASR (response in silence). Data are the mean \pm SEM,$n=8 /$ group. Two-way ANOVA with Bonferroni post hoc tests for $\boldsymbol{B}-\boldsymbol{F}$. ${ }^{*} p<0.05$, ${ }^{* *} p<0.01$, ${ }^{* * *} p<0.001$, ${ }^{* * * *} p<0.0001$. G, Ratio of the orientation (orient) and transition (trans) time in the presence of background WN (BWN) or series of clicks to the time in silence on rods of 20 or $15 \mathrm{~mm}$ diameters. The green line depicts the point when the ratio is 1 (no change in measured times with/without sound). Data are the mean \pm SEM (control, $n=4 ;$; Neurod1cK0,n=3). Statistical significance determined using two-way ANOVA with Sidak's multiple-comparison test. ${ }^{*} p<0.05$, ${ }^{* *} p<0.01$.

ular sensory epithelia previously only shown in certain neurotrophin mutants (Tessarollo et al., 2004) and Foxg1 mutant mice (Pauley et al., 2006). We tested whether the motor coordination of NeurodlcKO can be affected by acoustic stimulation. We conducted two different experiments, one with continuous
WN at an $80 \mathrm{~dB}$ SPL intensity, and another with a $600 \mathrm{~ms}$ series of clicks with a changing frequency of spike occurrence at an intensity of $70 \mathrm{~dB}$ SPL. We measured turning time (time taken to perform an $180^{\circ}$ turn from the starting position) and transition time on static rods of two different diameters. WN had a negative 


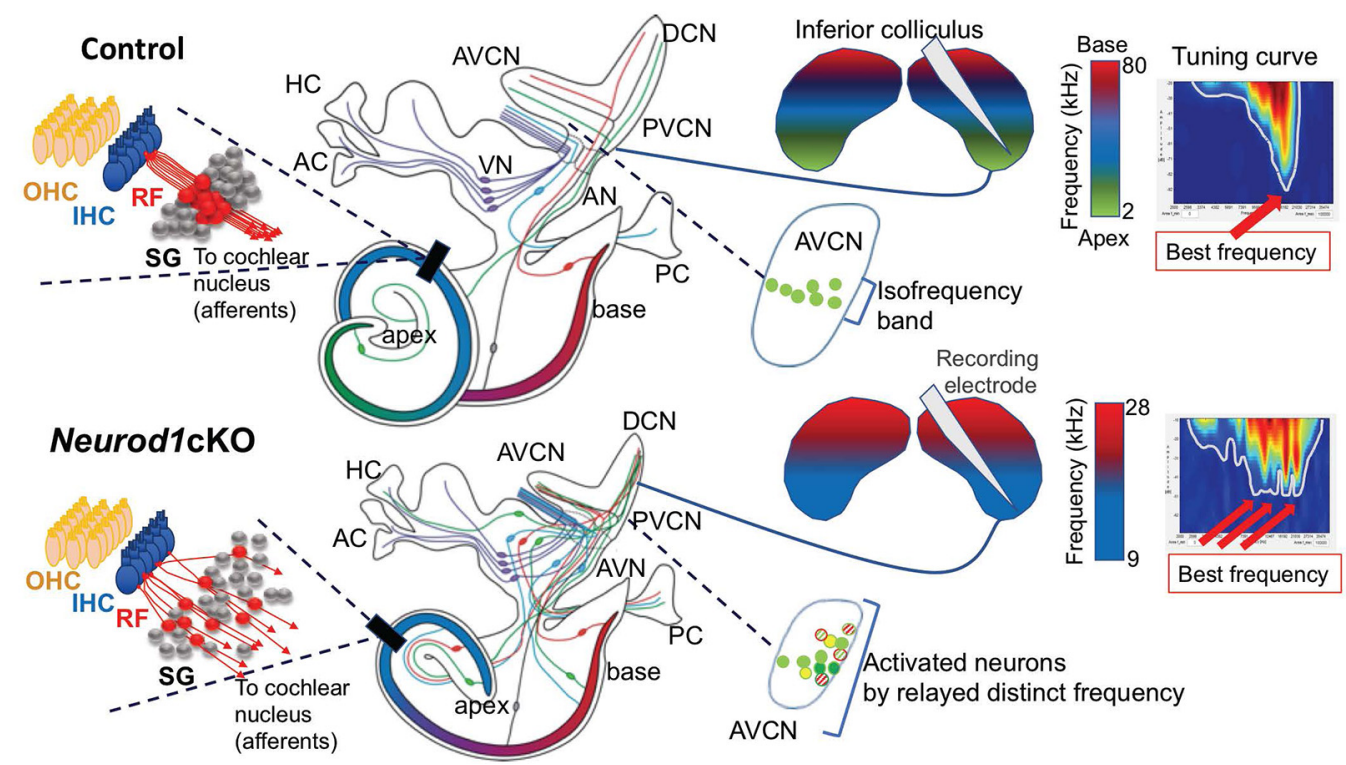

Figure 13. Model of tonotopic phenotype in Neurod 1cKO. In control mice, spiral ganglion afferent neurons (SGN) are radially connected with a single ending to IHCs (10 - 20 neurons/1 IHC). IHCS at different parts of the cochlea are stimulated by different frequencies, forming a tonotopic map with high frequencies at the base and low frequencies at the apex. The auditory system is tonotopically organized at all levels including the auditory nerve. The $\mathrm{CN}$ is the first target of the auditory nerve. SGN axons project centrally into the AVCN, relaying distinct frequencies within their corresponding isofrequency band. The IC is a major center for the integration of auditory sensory information from ascending pathways, including the pathway from the CN. The tuning curve of each IC unit represents a combination of the frequency and amplitude that evokes a response. At one frequency, the best frequency, sensitivity is highest. In Neurod $7 \mathrm{KK} 0$ mutants, the SG is disorganized, with aberrant innervation and a reduced number of neurons, many of which are displaced in the spiro-vestibular ganglion. There is little cochleotopic organization of afferents to the organ of Corti, within the auditory nerve and in the CN. SG afferents relaying specific frequencies target more AVCN cells outside the isofrequency band. Characteristics of IC neurons are altered with an enlarged frequency range and higher excitatory thresholds. The tuning characteristics of IC neurons are broad with multiple peaks of "best frequency". AC, Anterior crista; $A N$, auditory nerve; $\mathrm{HC}$, horizontal crista; $\mathrm{PC}$, posterior crista; $\mathrm{RF}$, radial fibers; $\mathrm{VG}$, vestibular ganglion; $\mathrm{VN}$, vestibular nerve.

effect on the transition time of Neurod1cKO mice on both rods (Fig. 12G). However, the train of clicks with changing frequency of occurrence had a lower impact on the mutant mice than on the controls. These results demonstrate that Neurod1 deletion affected the processing of vestibular information during sound exposure. Although we cannot exclude the possibility that the altered motor behavior of Neurod1cKO mutants was the result of their increased sound sensitivity to WN exposure, our data indicate that the vestibular and auditory systems may be functionally interconnected. The possibility of an interconnection is supported by the observed shared central projections and distribution of SG neurons in the vestibular ganglion and their projection to both the cochlea and vestibular organs.

\section{Discussion}

The formation of a neural map of the sensory periphery is an essential feature of all sensory systems. Understanding the mechanisms of formation, plasticity, and wiring of the sensory maps in both neonatal and adult states is a major endeavor in neuroscience. Sensory maps of the retina, somatosensory space, and cochlea show plastic reorganization of central map properties as a result of loss or modifications of specific features, for example filling in lost fingers or expanding size of somatosensory maps according to their use (Buonomano and Merzenich, 1998; Zhou and Merzenich, 2008), filling in lost areas of the retina (Baker et al., 2005; Keck et al., 2008), and even providing some hearing after destruction of SG neurons (Chambers et al., 2016; Wang, 2016). In contrast to all these data on ontogenetic and adult map effects, for the first time we succeeded to eliminate auditory primary map formation during development altogether. We genetically engineered a new mouse model lacking cochleotopic projections to the $\mathrm{CN}$ and tonotopic segregation in the ear. Using this new model, we have explored the ability of second-order neurons projecting from the $\mathrm{CN}$ to the IC to self-organize a secondary frequency map in the absence of a refined cochleotopic map. To the best of our knowledge, this is the first time that a single gene mutation results in physiologically uncorrectable primary map mistakes of both the peripheral and central innervation.

We show that genetically induced abnormal inner ear development results in a shorter cochlea, miswired and displaced SG neurons, and reduced spatially unsegregated central axons of SG neurons. The SG-to-CN afferent projections are represented by widely ramifying fibers that mostly reach the ventral part of the $\mathrm{CN}$ (Fig. 8). The tonotopic order of SG projections to all three $\mathrm{CN}$ subdivisions and their precise parallel fiber organization into isofrequency bands are largely absent in Neurod 1cKO. The processing of the limited frequency range of the periphery and $\mathrm{CN}$ is expanded across the entire IC (Fig. 13, schematic). These alterations of cochlear mapping onto the $\mathrm{CN}$ and $\mathrm{CN}$ onto the IC result in altered tuning characteristics of IC neurons in Neurod1cKO mutants, including an enlarged frequency range, higher excitatory thresholds, and worsened tuning capabilities. In association with the broad and multipeaked tuning curves of IC units, lower thresholds of startle responses to pure tones in mutants were measured, suggesting that mutants apparently perceive noiselike stimuli rather than pure tones. Modifications in intensity coding in the IC of Neurod $1 \mathrm{cKO}$ mutants indicate a central reorganization of the tuning properties of the auditory system with changes in the balance of inhibition and excitation as a response to the peripheral auditory deficiency. Furthermore, increased spontaneous activity of IC units in Neurod1cKO suggests hypersensitivity to sound in the central auditory pathways. Thus, our data provide evidence that a disorganized primary tonotopic auditory map leads to higher-order tono- 
topic information processing problems in the IC that are not self-correcting as seems to be the case for simple intensity distortions (Pelgrim et al., 2018).

Central reorganization of the auditory system has been previously demonstrated in studies using noise exposure or other cochlear damage protocols (Kandler et al., 2009; Harrison, 2016). However, unique to our study are the consequences of simultaneously disorganizing and compressing the primary sensory projection map onto the precise tonotopic organization of the central auditory pathways. Our data are in line with recent evidence from the $\mathrm{CN}$ and primary sound localization circuits that precise tonotopy depends on refinement at the subcellular and circuit level (summarized by Kandler et al., 2009; Clause et al., 2014). The smaller size of the $\mathrm{CN}$ and reduced cochlear nuclear circuits have been reported in genetically produced mutations, blocking synaptic transmission from hair cells and/or activation of SG neurons, and in cochlear ablation studies (Rubel and Fritzsch, 2002; Seal et al., 2008; Tritsch and Bergles, 2010; Tritsch et al., 2010); however, although reduced, the cochlear nuclear circuits that are established in these animals maintain their normal topographic organization patterns (Cao et al., 2008; Wright et al., 2014). This indicates that the reduction in the size of the $\mathrm{CN}$ of our Neurod1cKO mutant might only have a minimal contribution to the observed differences in the topographic disorganization of the $\mathrm{CN}$ and the responses of IC neurons, including wide and multipeaked tuning curves. Minor distortions of neuroanatomical mapping to the $\mathrm{CN}$ have been reported before, such as distortions in the patterning of the SG axons (Yang et al., 2017), and distortions of cochleotopic projections in Npr2 mutants ( $\mathrm{Lu}$ et al., 2014). Analysis of Hoxa2 mutants imply that even relatively minor alterations in isofrequency mapping have behavioral consequences (Karmakar et al., 2017). Compared with these minor changes of topological projections, our profoundly disorganized and truncated cochleotopic mapping implies that NEUROD1 may specify some navigational cues in SG neurons that read diffusible gradients mediated by specific Wnt signals in the dorsal hindbrain (Yang et al., 2017), substrate cues (Gu et al., 2003; Lu et al., 2014), and physiological activity (Kandler et al., 2009; Marrs and Spirou, 2012; Elliott et al., 2015) to establish the cochleotopic map onto the CN. NEUROD1 variably regulates, directly or indirectly, 3000 genes, including nearly all frizzled class receptor (Fzd) genes (Pataskar et al., 2016) relevant for Wnt signaling (Dijksterhuis et al., 2014). Without NEUROD1, spiral ganglia afferent fibers will experience expression changes in many genes possibly involved in neurite guidance. For example, alterations in local Fzd receptors could provide further mechanistic insights into the distorted mapping of Neurod 1cKO SG neurons. In addition, bHLH proteins interact with Gata zinc-finger proteins (Rawnsley et al., 2013). There is an interesting resemblance of phenotypes between a delayed Gata3 conditional KO (Duncan and Fritzsch, 2013) and our Neurod1cKO mutant. Both mutants have decreased number and misplaced SG neurons, and display incorrect patterning within the $\mathrm{CN}$. However, the blurring of the boundaries between spiral and vestibular ganglion neurons with SVG formation and multiple branches within the inner ear represents a unique feature of our Neurod1cKO mutant. Although some afferents can still reach the $\mathrm{CN}$, their inability to navigate properly results in reduced and disorganized coverage of the $\mathrm{CN}$ in the NeurodlcKO mutant. In particular, the well known regulation of neurotrophin receptors Ntrk2 (TrkB) and Ntrk3 (TrkC) by NEUROD1 (Kim et al., 2001) may regulate the branching of afferents previously described using neurotrophin and neurotrophin receptor deletions (Fariñas et al., 2001; Fritzsch et al., 2016b), and misexpression (Tessarollo et al., 2004; Yang et al., 2011). Neurod1 mutation might also affect the central projections, as previously described in Neurotrophin3 mutants, which use BDNF/Ntrk2 signaling to expand into the $\mathrm{CN}$ after a partial loss of basal turn SG neurons (Fritzsch et al., 1997). The expanded branching of afferents along the cochlea in our mutant, with basal turn SG neurons projecting to the apex (and vice versa; Fig. 6), generates broad mapping of large areas of the cochlea to only parts of the ventral CN (Fig. 13, summary) instead of a point to point connection as in control animals (Muniak et al., 2016).

The connectional reasons for the occurrence of the unusual multipeak broad tuning curves in the IC of our Neurod1cKO mutants are yet to be specified. Nevertheless, our data show that the peripheral deficit in sound encoding cannot be compensated up to the level of the IC. The tuning curves of single units in the IC of control mice have a characteristic $\mathrm{V}$-shape with one peak and thus one best frequency. In the Neurod1cKO, there are either two-peak or multipeak tuning curves, suggesting multiple frequency inputs. Where this integration of inputs occurs must be shown in further experiments. Clearly, at the level of the cochlea, neurons of the SG are connected to more than one IHC as revealed by our dye tracing data, which show that single SG neurons reach multiple areas of the organ of Corti, for example a given neuron can reach both the base and apex (Fig. 6). This would explain the multipeaked tuning curves in the IC. However, we also cannot exclude more SG neurons with different frequency tuning being connected to several $\mathrm{CN}$ neurons as afferents are not segregated into isofrequency bands in the CN (Fig. 8).

In conclusion, our data demonstrate that the physiological tonotopic properties of the NeurodlcKO auditory system are changed at all levels investigated. It remains unclear how exactly the frequency presentation distortion of the cochlea onto the cochlear nuclei relates to these property changes and other well known plastic changes in the cortical auditory system (Harrison, 2016). Our behavioral data show that the startle response is affected and that the cross-innervation of vestibular end-organs affects complex vestibular functions after sound disturbance. Future work is needed to establish how tonotopic distortions affect space map formation (Syka, 2002; Eggermont, 2017) and cortical information processing (Kral et al., 2016) that could benefit cochlear implant treatment (Harrison, 2016). Using ouabain in our mouse model should allow to demonstrate that the reported recovery after afferent ablation (Chambers et al., 2016) relies on a developmental stabilization of tonotopic map features which we have disrupted here.

\section{References}

Baker CI, Peli E, Knouf N, Kanwisher NG (2005) Reorganization of visual processing in macular degeneration. J Neurosci 25:614-618. CrossRef Medline

Bohuslavova R, Dodd N, Macova I, Chumak T, Horak M, Syka J, Fritzsch B, Pavlinkova G (2017) Pax2-Islet1 transgenic mice are hyperactive and have altered cerebellar foliation. Mol Neurobiol 54:1352-1368. CrossRef Medline

Buonomano DV, Merzenich MM (1998) Cortical plasticity: from synapses to maps. Annu Rev Neurosci 21:149-186. CrossRef Medline

Buran BN, Strenzke N, Neef A, Gundelfinger ED, Moser T, Liberman MC (2010) Onset coding is degraded in auditory nerve fibers from mutant mice lacking synaptic ribbons. J Neurosci 30:7587-7597. CrossRef Medline

Bures Z, Grécová J, Popelár J, Syka J (2010) Noise exposure during early development impairs the processing of sound intensity in adult rats. Eur J Neurosci 32:155-164. CrossRef Medline

Cao XJ, McGinley MJ, Oertel D (2008) Connections and synaptic function in the posteroventral cochlear nucleus of deaf jerker mice. J Comp Neurol 510:297-308. CrossRef Medline 
Chagnaud BP, Engelmann J, Fritzsch B, Glover JC, Straka H (2017) Sensing external and self-motion with hair cells, a comparison of the lateral line and vestibular systems from a developmental and evolutionary perspective. Brain Behav Evol 90:98-116. CrossRef Medline

Chambers AR, Resnik J, Yuan Y, Whitton JP, Edge AS, Liberman MC, Polley DB (2016) Central gain restores auditory processing following nearcomplete cochlear denervation. Neuron 89:867-879. CrossRef Medline

Chumak T, Bohuslavova R, Macova I, Dodd N, Buckiova D, Fritzsch B, Syka J, Pavlinkova G (2016) Deterioration of the medial olivocochlear efferent system accelerates age-related hearing loss in Pax2-Isl1 transgenic mice. Mol Neurobiol 53:2368-2383. CrossRef Medline

Clause A, Kim G, Sonntag M, Weisz CJ, Vetter DE, Rúbsamen R, Kandler K (2014) The precise temporal pattern of prehearing spontaneous activity is necessary for tonotopic map refinement. Neuron 82:822-835. CrossRef Medline

Constantine-Paton M, Law MI (1978) Eye-specific termination bands in tecta of three-eyed frogs. Science 202:639-641. CrossRef Medline

Cramer KS, Gabriele ML (2014) Axon guidance in the auditory system: multiple functions of Eph receptors. Neuroscience 277:152-162. CrossRef Medline

Cruces-Solís H, Jing Z, Babaev O, Rubin J, Gür B, Krueger-Burg D, Strenzke N, de Hoz L (2018) Auditory midbrain coding of statistical learning that results from discontinuous sensory stimulation. PLoS Biol 16:e2005114. CrossRef Medline

Deacon R (2013) Measuring motor coordination in mice. J Vis Exp 75: e2609. CrossRef Medline

Dijksterhuis JP, Petersen J, Schulte G (2014) WNT/Frizzled signalling: receptor-ligand selectivity with focus on FZD-G protein signalling and its physiological relevance: IUPHAR review 3. Br J Pharmacol 171:11951209. CrossRef Medline

Duncan JS, Fritzsch B (2013) Continued expression of GATA3 is necessary for cochlear neurosensory development. PLoS One 8:e62046. CrossRef Medline

Dvorakova M, Jahan I, Macova I, Chumak T, Bohuslavova R, Syka J, Fritzsch B, Pavlinkova G (2016) Incomplete and delayed Sox2 deletion defines residual ear neurosensory development and maintenance. Sci Rep 6:38253. CrossRef Medline

Eggermont JJ (2017) Acquired hearing loss and brain plasticity. Hear Res 343:176-190. CrossRef Medline

Elliott KL, Houston DW, Fritzsch B (2015) Sensory afferent segregation in three-eared frogs resemble the dominance columns observed in threeeyed frogs. Sci Rep 5:8338. CrossRef Medline

Fariñas I, Jones KR, Tessarollo L, Vigers AJ, Huang E, Kirstein M, de Caprona DC, Coppola V, Backus C, Reichardt LF, Fritzsch B (2001) Spatial shaping of cochlear innervation by temporally regulated neurotrophin expression. J Neurosci 21:6170-6180. CrossRef Medline

Fritzsch B, Elliott KL (2017) Evolution and development of the inner ear efferent system: transforming a motor neuron population to connect to the most unusual motor protein via ancient nicotinic receptors. Front Cell Neurosci 11:114. CrossRef Medline

Fritzsch B, Fariñas I, Reichardt LF (1997) Lack of neurotrophin 3 causes losses of both classes of spiral ganglion neurons in the cochlea in a regionspecific fashion. J Neurosci 17:6213-6225. CrossRef Medline

Fritzsch B, Pauley S, Feng F, Matei V, Nichols DH (2006) The molecular and developmental basis of the evolution of the vertebrate auditory system. Int J Comp Psychol 19:1-25.

Fritzsch B, Dillard M, Lavado A, Harvey NL, Jahan I (2010) Canal cristae growth and fiber extension to the outer hair cells of the mouse ear require Proxl activity. PloS One 5:e9377. CrossRef Medline

Fritzsch B, Duncan JS, Kersigo J, Gray B, Elliott KL (2016a) Neuroanatomical tracing techniques in the ear: history, state of the art, and future developments. In: Auditory and vestibular research: methods and protocols (Sokolowski B, ed), pp. 243-262. New York: Springer.

Fritzsch B, Kersigo J, Yang T, Jahan I, Pan N (2016b) Neurotrophic factor function during ear development: expression changes define critical phases for neuronal viability. In: The primary auditory neurons of the mammalian cochlea, pp. 49-84. New York: Springer.

Goebbels S, Bode U, Pieper A, Funfschilling U, Schwab MH, Nave KA (2005) Cre/loxP-mediated inactivation of the bHLH transcription factor gene NeuroD/BETA2. Genesis 42:247-252. CrossRef Medline

Gogos JA, Osborne J, Nemes A, Mendelsohn M, Axel R (2000) Genetic ab- lation and restoration of the olfactory topographic map. Cell 103:609620. CrossRef Medline

Goodhill GJ (2007) Contributions of theoretical modeling to the understanding of neural map development. Neuron 56:301-311. CrossRef Medline

Goodrich LV (2016) Early development of the spiral ganglion. In: The primary auditory neurons of the mammalian cochlea, pp. 11-48. New York: Springer.

Gu C, Rodriguez ER, Reimert DV, Shu T, Fritzsch B, Richards LJ, Kolodkin AL, Ginty DD (2003) Neuropilin-1 conveys semaphorin and VEGF signaling during neural and cardiovascular development. Dev Cell 5:45-57. CrossRef Medline

Gurung B, Fritzsch B (2004) Time course of embryonic midbrain and thalamic auditory connection development in mice as revealed by carbocyanine dye tracing. J Comp Neurol 479:309-327. CrossRef Medline

Harrison RV (2016) Biologic development of the auditory system from periphery to cortex. In: Comprehensive handbook of pediatric audiology, p. 23. San Diego: Plural.

Hickox AE, Liberman MC (2014) Is noise-induced cochlear neuropathy key to the generation of hyperacusis or tinnitus? J Neurophysiol 111:552-564. CrossRef Medline

Hubel DH, Wiesel TN, LeVay S (1977) Plasticity of ocular dominance columns in monkey striate cortex. Philos Trans R Soc Lond B Biol Sci 278: 377-409. CrossRef Medline

Huberman AD, Feller MB, Chapman B (2008) Mechanisms underlying development of visual maps and receptive fields. Annu Rev Neurosci 31: 479-509. CrossRef Medline

Jahan I, Kersigo J, Pan N, Fritzsch B (2010a) Neurod1 regulates survival and formation of connections in mouse ear and brain. Cell Tissue Res 341:95110. CrossRef Medline

Jahan I, Pan N, Kersigo J, Fritzsch B (2010b) Neurod1 suppresses hair cell differentiation in ear ganglia and regulates hair cell subtype development in the cochlea. PLoS One 5:e11661. CrossRef Medline

Kandler K, Clause A, Noh J (2009) Tonotopic reorganization of developing auditory brainstem circuits. Nat Neurosci 12:711-717. CrossRef Medline

Karmakar K, Narita Y, Fadok J, Ducret S, Loche A, Kitazawa T, Genoud C, Di Meglio T, Thierry R, Bacelo J, Lüthi A, Rijli FM (2017) Hox2 genes are required for tonotopic map precision and sound discrimination in the mouse auditory brainstem. Cell Rep 18:185-197. CrossRef Medline

Keck T, Mrsic-Flogel TD, Vaz Afonso M, Eysel UT, Bonhoeffer T, Hübener M (2008) Massive restructuring of neuronal circuits during functional reorganization of adult visual cortex. Nat Neurosci 11:1162-1167. CrossRef Medline

Kim WY, Fritzsch B, Serls A, Bakel LA, Huang EJ, Reichardt LF, Barth DS, Lee JE (2001) NeuroD-null mice are deaf due to a severe loss of the inner ear sensory neurons during development. Development 128:417-426. Medline

Krahe R, Maler L (2014) Neural maps in the electrosensory system of weakly electric fish. Curr Opin Neurobiol 24:13-21. CrossRef Medline

Kral A, Kronenberger WG, Pisoni DB, O’Donoghue GM (2016) Neurocognitive factors in sensory restoration of early deafness: a connectome model. Lancet Neurol 15:610-621. CrossRef Medline

Liu M, Pereira FA, Price SD, Chu MJ, Shope C, Himes D, Eatock RA, Brownell WE, Lysakowski A, Tsai MJ (2000) Essential role of BETA2/NeuroD1 in development of the vestibular and auditory systems. Genes Dev 14:2839_ 2854. CrossRef Medline

Lu CC, Cao XJ, Wright S, Ma L, Oertel D, Goodrich LV (2014) Mutation of Npr2 leads to blurred tonotopic organization of central auditory circuits in mice. PLoS Genetics 10:e1004823. CrossRef Medline

Mao Y, Reiprich S, Wegner M, Fritzsch B (2014) Targeted deletion of Sox10 by Wnt1-cre defects neuronal migration and projection in the mouse inner ear. PloS One 9:e94580. CrossRef Medline

Marrs GS, Spirou GA (2012) Embryonic assembly of auditory circuits: spiral ganglion and brainstem. J Physiol 590:2391-2408. CrossRef Medline

Martin MR, Rickets C (1981) Histogenesis of the cochlear nucleus of the mouse. J Comp Neurol 197:169-184. CrossRef Medline

Mombaerts P (1999) Molecular biology of odorant receptors in vertebrates. Annu Rev Neurosci 22:487-509. CrossRef Medline

Müller M, von Hünerbein K, Hoidis S, Smolders JW (2005) A physiological place-frequency map of the cochlea in the CBA/J mouse. Hear Res 202: 63-73. CrossRef Medline

Muniak MA, Rivas A, Montey KL, May BJ, Francis HW, Ryugo DK (2013) 
3D model of frequency representation in the cochlear nucleus of the CBA/J mouse. J Comp Neurol 521:1510-1532. CrossRef Medline

Muniak MA, Connelly CJ, Suthakar K, Milinkeviciute G, Ayeni FE, Ryugo DK (2016) Central projections of spiral ganglion neurons. In: The primary auditory neurons of the mammalian cochlea, pp. 157-190. New York: Springer.

Pataskar A, Jung J, Smialowski P, Noack F, Calegari F, Straub T, Tiwari VK (2016) NeuroD1 reprograms chromatin and transcription factor landscapes to induce the neuronal program. EMBO J 35:24-45. CrossRef Medline

Pauley S, Lai E, Fritzsch B (2006) Foxg1 is required for morphogenesis and histogenesis of the mammalian inner ear. Dev Dyn 235:2470-2482. CrossRef Medline

Pelgrim M, Yamanbaeva G, Reisinger E, Strenzke N (2018) Sound encoding in the inferior colliculus of otoferlin Ile515Thr mutant mice. Laryngorhinootologie 97:S230-S231. CrossRef

Penfield W, Boldrey E (1937) Somatic motor and sensory representation in the cerebral cortex of man as studied by electrical stimulation. Brain 60:389-443. CrossRef

Rawnsley DR, Xiao J, Lee JS, Liu X, Mericko-Ishizuka P, Kumar V, He J, Basu A, Lu M, Lynn FC, Pack M, Gasa R, Kahn ML (2013) The transcription factor atonal homolog 8 regulates Gata4 and Friend of Gata-2 during vertebrate development. J Biol Chem 288:24429-24440. CrossRef Medline

Renier N, Dominici C, Erzurumlu RS, Kratochwil CF, Rijli FM, Gaspar P, Chédotal A (2017) A mutant with bilateral whisker to barrel inputs unveils somatosensory mapping rules in the cerebral cortex. eLife 6:e23494. CrossRef Medline

Rubel EW, Fritzsch B (2002) Auditory system development: primary auditory neurons and their targets. Annu Rev Neurosci 25:51-101. CrossRef Medline

Seal RP, Akil O, Yi E, Weber CM, Grant L, Yoo J, Clause A, Kandler K, Noebels JL, Glowatzki E, Lustig LR, Edwards RH (2008) Sensorineural deafness and seizures in mice lacking vesicular glutamate transporter 3. Neuron 57:263-275. CrossRef Medline

Sienknecht UJ, Köppl C, Fritzsch B (2014) Evolution and development of hair cell polarity and efferent function in the inner ear. Brain Behav Evol 83:150-161. CrossRef Medline
Simmons D, Duncan J, de Caprona DC, Fritzsch B (2011) Development of the inner ear efferent system. In: Auditory and vestibular efferents, pp. 187-216. New York: Springer.

Sperry RW (1963) Chemoaffinity in the orderly growth of nerve fiber patterns and connections. Proc Natl Acad Sci U S A 50:703-710. CrossRef Medline

Syka J (2002) Plastic changes in the central auditory system after hearing loss, restoration of function, and during learning. Physiol Rev 82:601636. CrossRef Medline

Tessarollo L, Coppola V, Fritzsch B (2004) NT-3 replacement with brainderived neurotrophic factor redirects vestibular nerve fibers to the cochlea. J Neurosci 24:2575-2584. CrossRef Medline

Tomková M, Tomek J, Novák O, Zelenka O, Syka J, Brom C (2015) Formation and disruption of tonotopy in a large-scale model of the auditory cortex. J Comput Neurosci 39:131-153. CrossRef Medline

Tritsch NX, Bergles DE (2010) Developmental regulation of spontaneous activity in the mammalian cochlea. J Neurosci 30:1539-1550. CrossRef Medline

Tritsch NX, Rodríguez-Contreras A, Crins TT, Wang HC, Borst JG, Bergles DE (2010) Calcium action potentials in hair cells pattern auditory neuron activity before hearing onset. Nat Neurosci 13:1050-1052. CrossRef Medline

Wang X (2016) The yin and yang of auditory nerve damage. Neuron 89 : 680-682. CrossRef Medline

Wright S, Hwang Y, Oertel D (2014) Synaptic transmission between end bulbs of held and bushy cells in the cochlear nucleus of mice with a mutation in otoferlin. J Neurophysiol 112:3173-3188. CrossRef Medline

Yang T, Kersigo J, Jahan I, Pan N, Fritzsch B (2011) The molecular basis of making spiral ganglion neurons and connecting them to hair cells of the organ of corti. Hear Res 278:21-33. CrossRef Medline

Yang T, Kersigo J, Wu S, Fritzsch B, Bassuk AG (2017) Pricklel regulates neurite outgrowth of apical spiral ganglion neurons but not hair cell polarity in the murine cochlea. PloS One 12:e0183773. CrossRef Medline

Zhou X, Merzenich MM (2008) Enduring effects of early structured noise exposure on temporal modulation in the primary auditory cortex. Proc Natl Acad Sci U S A 105:4423-4428. CrossRef Medline 